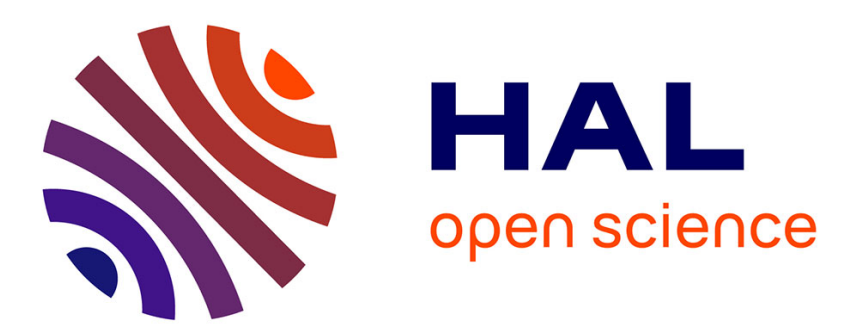

\title{
Differing impacts of resolution changes in latitude and longitude on the midlatitudes in the LMDZ atmospheric GCM
}

\author{
Virginie Guemas, Francis Codron
}

\section{> To cite this version:}

Virginie Guemas, Francis Codron. Differing impacts of resolution changes in latitude and longitude on the midlatitudes in the LMDZ atmospheric GCM. Journal of Climate, 2011, 24 (22), pp.5831-5849. 10.1175/2011JCLI4093.1. hal-01119137

\author{
HAL Id: hal-01119137 \\ https://hal.science/hal-01119137
}

Submitted on 21 Feb 2015

HAL is a multi-disciplinary open access archive for the deposit and dissemination of scientific research documents, whether they are published or not. The documents may come from teaching and research institutions in France or abroad, or from public or private research centers.
L'archive ouverte pluridisciplinaire HAL, est destinée au dépôt et à la diffusion de documents scientifiques de niveau recherche, publiés ou non, émanant des établissements d'enseignement et de recherche français ou étrangers, des laboratoires publics ou privés. 


\title{
Differing Impacts of Resolution Changes in Latitude and Longitude on the Midlatitudes in the LMDZ Atmospheric GCM
}

\author{
VIRGINIE GUEMAS* AND FRANCIS CODRON \\ Laboratoire de Météorologie Dynamique, CNRS, Université Pierre et Marie Curie, Paris, France
}

(Manuscript received 22 September 2010, in final form 27 April 2011)

\begin{abstract}
This article examines the sensitivity of the Laboratoire de Météorologie Dynamique Model with Zoom Capability (LMDZ), a gridpoint atmospheric GCM, to changes in the resolution in latitude and longitude, focusing on the midlatitudes. In a series of dynamical core experiments, increasing the resolution in latitude leads to a poleward shift of the jet, which also becomes less baroclinic, while the maximum eddy variance decreases. The distribution of the jet positions in time also becomes wider. On the contrary, when the resolution increases in longitude, the position and structure of the jet remain almost identical, except for a small equatorward shift tendency. An increase in eddy heat flux is compensated by a strengthening of the Ferrel cell. The source of these distinct behaviors is then explored in constrained experiments in which the zonal-mean zonal wind is constrained toward the same reference state while the resolution varies. While the low-level wave sources always increase with resolution in that case, there is also enhanced poleward propagation when increasing the resolution in longitude, preventing the jet shift. The diverse impacts on the midlatitude dynamics hold when using the full GCM in a realistic setting, either forced by observed SSTs or coupled to an ocean model.
\end{abstract}

\section{Introduction}

\section{a. Sensitivity of the simulated climate to the model resolution}

During the last two decades, the rapid advances in computer power have allowed incorporation of new components of the climate system and to represent more and more physical processes in global climate models. These advances have also allowed a nearly doubling of the model spatial resolution between each Intergovernmental Panel on Climate Change (IPCC) assessment report. This rapid increase in resolution elicited early studies aiming to quantify the improvements brought on by such a refinement in a number of atmospheric general circulation models (AGCMs) and to determine the "best" resolution to optimally represent climate processes.

\footnotetext{
* Current affiliation: Institut Català de Ciències del Clima, Barcelona, Spain.

Corresponding author address: Virginie Guemas, Institut Català de Ciències del Clima, Carrer del Doctor Trueta 203, 08005 Barcelona, Spain.

E-mail:vguemas@ic3.cat
}

Significant impacts were found on a range of climate features: zonal winds (Boville 1991; Boyle 1993; Déqué et al. 1994), eddy kinetic energy (EKE) and heat and momentum fluxes (Boville 1991; Boyle 1993), stationary eddies (Chen and Tribbia 1993), cloud cover and precipitation (Kiehl and Williamson 1991; Déqué et al. 1994; Phillips et al. 1995), and ozone concentration (Déqué et al. 1994).

While the first studies could not highlight a resolution better than the others (Boer and Lazare 1988), a general improvement of the simulation was found by Boville (1991), Kiehl and Williamson (1991), and Williamson et al. (1995) from the T21 to the T63 grids. The numerical solutions were found to converge at the T42 (Boyle 1993) and T63 (Williamson et al. 1995; Boer and Denis 1997) resolutions for mean fields, such as the zonal-mean wind and temperature, the meridional circulation, the eddy statistics, the vertical motions, the moisture patterns, and the cloud fraction. Later studies at higher resolutions showed that while some features of the models tend to converge at commonly used resolution (surface heat and momentum fluxes, equatorial waves), other ones keep evolving even at very high resolutions. These convergence properties are highly model dependent, with no convergence at the N144 resolution for moisture and precipitation in the third Hadley 
Centre Atmosphere Model (HadAM3) (Pope and Stratton 2002); at the T319 resolution for eddy kinetic energy, midtroposphere geopotential height, zonal-mean wind and temperature, and meridional circulation in the European Centre for Medium-Range Weather Forecasts (ECMWF) model (Branković and Gregory 2001); and at the T340 resolution for sea level pressure, cloud fraction, and eddy heat and momentum fluxes in the National Center for Atmospheric Research Community Climate Model, version 2 (CCM2) (Williamson 1999; Hack et al. 2006; Williamson 2008). In the ECHAM4 model from the Max Planck Institute for Meteorology, the convergence was found to depend on the vertical resolution (Roeckner et al. 2006).

\section{b. The midlatitude atmospheric circulation}

Focusing on the midlatitude dynamics, a better agreement can be found between the different models. An increase in horizontal resolution generally leads to a tropospheric warming and a poleward shift of the westerly jets (Boyle 1993; Williamson et al. 1995; Hack et al. 2006; Stendel and Roeckner 2006; Roeckner et al. 2006; Wan et al. 2008; Déqué et al. 1994; Boer and Denis 1997; Stratton 1999; Pope and Stratton 2002). In the Geophysical Fluid Dynamics Laboratory (GFDL) model (Held and Phillipps 1993; Hamilton et al. 1995), the midlatitudinal jet intensifies but does not shift poleward.

These changes in the zonal-mean wind are accompanied by an increase in eddy kinetic energy and eddy momentum fluxes with resolution (Boyle 1993; Held and Phillipps 1993; Williamson et al. 1995; Stratton 1999). At low resolutions (R15-R30), Held and Phillipps (1993) highlight the major role of the increase in latitudinal resolution rather than longitudinal resolution in explaining the increase in eddy momentum flux and hence zonal winds. The sensitivity to the latitudinal resolution reflects the need to resolve small scales generated in the Rossby wave field, which are not resolved at low resolutions such as R15. Using the experimental setup proposed by Held and Suarez (1994), Wan et al. (2008) similarly obtained an increase in baroclinic wave amplitude (BWA) when increasing the resolution from T31 to T159 in the ECHAM5 dynamical core, associated with an increase in eddy heat flux, a warming of the midand high latitudes, and a weakening and poleward shift of the jet. On the contrary, Pope and Stratton (2002) attributed the poleward shift of the extratropical jets to changes in the hydrological cycle in the full HadAM3 model (N48-N144). In their dynamical core experiments following the Held and Suarez (1994) proposal, they also obtained an increase in transient eddy kinetic energy and an upper-tropospheric warming but no shift of the jet. Despite the agreement between a number of atmospheric models in showing a poleward shift of the jet when increasing the horizontal resolution, the mechanisms by which this shift occurs do not yet bring such a consensus.

\section{c. Aim of this study}

The aim of this paper is to document the impact of the horizontal resolution on the zonally averaged midlatitude atmospheric circulation in the Laboratoire de Météorologie Dynamique Model with Zoom Capability (LMDZ), which is the atmospheric component of L'Institut Pierre-Simon Laplace (IPSL) global climate model used for IPCC projections. To better explore the reasons for the changes, we use mostly idealized dynamical core experiments. Additionally, as an original approach, we analyze separately the impacts of changes in the resolution in latitude and in longitude, extending the idea of Held and Phillipps (1993) at higher resolutions. The more general impact of the resolution on the climate simulated by the full coupled model, its variability and sensitivity, were partially described in Marti et al. (2010) and will be the object of another study.

The paper is organized as follows: In section 2, we diagnose the different impacts of the latitudinal and longitudinal resolutions on the midlatitude atmospheric circulation in the full AGCM, in coupled mode or forced by observed SSTs. Section 3 demonstrates the major role of the dynamical core of version 4 of the LMDZ (LMDZ4) on these effects. Section 4 investigates the responsible processes by means of constrained experiments in which the zonal-mean jet is imposed. Sections 5 and 6 are dedicated to discussions and conclusions, respectively.

\section{Sensitivity of the full atmospheric model}

\section{a. The IPSL coupled general circulation model}

The IPSL (Marti et al. 2005, 2010) climate model is based on the coupled core formed by the LMDZ4 AGCM (Hourdin et al. 2006), the Océan Parallélisé (OPA) ocean general circulation model (OGCM) (Madec et al. 1997), the Louvain-la-Neuve Sea-Ice Model (LIM) (Fichefet and Morales Maqueda 1997, 1999), and the Organizing Carbon and Hydrology in Dynamic Ecosystems (ORCHIDEE) (Krinner et al. 1997) land surface model. The LMDZ4 component is briefly described in the following. Full details about the IPSL model are given in Marti et al. (2010).

\section{THE LMDZ4 ATMOSPHERIC MODEL}

The dynamical part of the LMDZ4 code is based on a finite-difference formulation of the primitive equations of meteorology. These equations are discretized on 
a) Coupled model

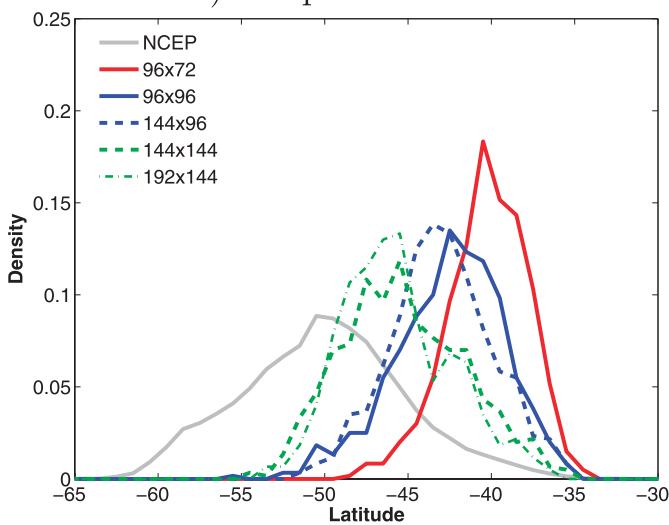

b) Atmosphere model

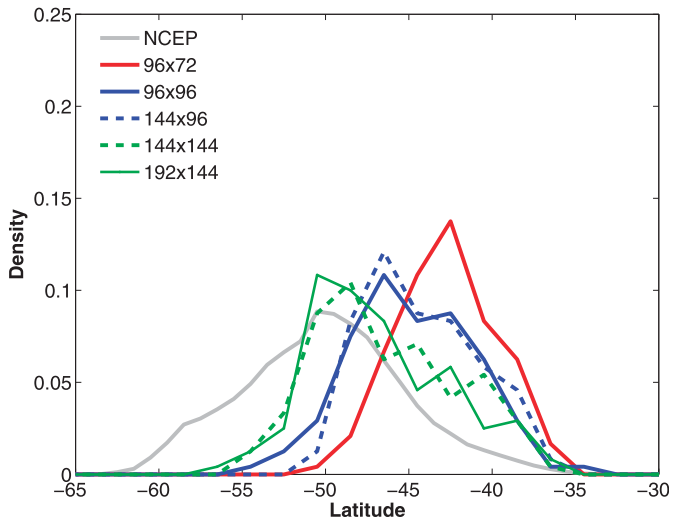

FIG. 1. Probability density function (PDF) of the Southern Hemisphere winter jet positions, computed as the latitude of the zonal-mean zonal wind maximum at the $850-\mathrm{hPa}$ level, (a) for the five coupled simulations run with the IPSL coupled model and described in section $2 \mathrm{~b}$ and (b) for the five atmosphere-only simulations run with the LMDZ4 model and described in section 2c. The PDF for the NCEP reanalysis is shown in gray.

the sphere in a staggered and longitude-latitude Arakawa C-grid (Kasahara 1977). The discretization insures numerical conservation of both enstrophy (square of the wind rotational) for barotropic flows, following Sadourny (1975a,b), and the axi-symmetric component of angular momentum. For latitudes poleward of $60^{\circ}$ in both hemispheres, a longitudinal filter is applied to limit the effective resolution to that at $60^{\circ}$. Horizontal dissipation is added to the dynamical equations. Based on an iterated Laplacian, it is designed so as to represent properly the pumping of enstrophy at the cutoff scale. The time step is bounded by a Courant-Friedrichs-Lewy (CFL) criterion of the fastest gravity modes. The time integration uses a leapfrog scheme with a periodic predictor-corrector time step. The time step is of $2 \mathrm{~h}$ for the radiation scheme, $30 \mathrm{~min}$ for physics, and $3 \mathrm{~min}$ in the standard resolution $(96 \times 72)$ for the dynamics. On the vertical, the model uses a classical hybrid $\sigma-p$ coordinate and has 19 layers with the first four layers in the first kilometer above surface, a mean resolution of about $2 \mathrm{~km}$ between 2 and $20 \mathrm{~km}$ and four layers above $20 \mathrm{~km}$. Vapor and liquid water are advected with a monotonic second-order finite volume scheme (Van Leer 1977; Hourdin and Armengaud 1999).

\section{b. Position of the jet in the coupled model}

Five coupled simulations were run with the IPSL coupled model changing the LMDZ4 horizontal resolution, its dynamical time step to respect the CFL criterion, and the horizontal dissipation scale for consistency; the other parameters being kept constant. The selected resolutions are $96 \times 72,96 \times 96,144 \times 96$, $144 \times 144$, and $192 \times 144$, where the first and second numbers correspond to the longitudinal and latitudinal number of grid points, respectively. These simulations last 50 yr. Note that the $96 \times 96$ and $144 \times 144$ resolutions are isotropics at $60^{\circ}$, while the $144 \times 72$ resolution is isotropic at the equator.

In the rest of this article, all the figures presenting some statistics on a consistent set of experiments will follow the same color code. Each selected color corresponds to one latitudinal resolution (green $=144$, blue $=96$, red $=72$, black $=48)$, while each selected line style and symbol corresponds to one longitudinal resolution (dasheddotted $/$ diamond $=48$, dotted/up triangle $=72$, continuous $/$ square $=96$, dashed $/$ circle $=144$, dashed-dotted $/$ down triangle $=192)$. When showing differences in some statistics between couples of simulation, a change in latitudinal resolution is plotted in red, while a change in longitudinal resolution is plotted in blue.

Figure 1a shows the distribution of the position of the Southern Hemisphere jet in each of the coupled simulations, as well as in the National Centers for Environmental Prediction (NCEP) reanalysis. The jet position is defined as the location of the zonal-mean zonal wind maximum at $850 \mathrm{hPa}$. An increase in the latitudinal resolution of the LMDZ4 atmospheric model is associated with a poleward shift and an increase in variance of the distribution. The mean position of the jet in the $96 \times 96(144 \times 144)$ simulation is poleward of its mean position in the $96 \times 72(144 \times 96)$ simulation by $2.27^{\circ}$ $\left(2.28^{\circ}\right)$. These shifts of the position are both significant at the $99 \%$ level ( $p$ value $<2.2 \times 10^{-16}$ ); we use for differences in the jet position a two-sided $t$ test with a Welch approximation of the degrees of freedom to account for the difference in variance of the two distributions. The variance of the jet position in the $96 \times 96$ $(144 \times 144)$ simulation is $1.82(1.15)$ times the one in the 
$96 \times 72(144 \times 96)$ simulation. These differences in variance are both significant at the $90 \%$ level using a Fisher test ( $p$ value $\simeq 10^{-13}$ and $p$ value $=0.083$, respectively). In the $96 \times 72$ simulation, which corresponds to the resolution used in the IPSL Coupled Model, version 4 (IPSL CM4) for the IPCC Fourth Assessment Report (AR4), the jet is far too equatorward relative to the NCEP data (by $9.8^{\circ}$ ). The IPSL CM4 stands among the worst coupled models used in the AR4 framework (Russell et al. 2006) regarding this aspect, together with the Goddard Institute for Space Studies Atmosphere-Ocean Model (GISS-AOM). Increasing the latitudinal resolution improves the representation of the midlatitudinal jet in the coupled model. However, even in the simulation with the highest resolution $(192 \times 144)$, the jet is still too equatorward by $4.36^{\circ}$. The variance of its position is also underestimated by a factor 2.24. An increase in the longitudinal resolution has a negligible effect on the jet position or its variance.

We can wonder if the impact of the horizontal resolution on the jet position comes from the dynamical core of the LMDZ4 atmospheric model, its physical parameterizations, or the interaction with the other components of the coupled model, such as the ocean, for example. To test the third hypothesis, we perform the same diagnoses in a corresponding set of atmosphereforced simulations.

\section{c. Position of the jet in the atmosphere-only simulations}

We now consider simulations run with varying horizontal resolution, dynamical time step, and horizontal dissipation scale as in the set of coupled simulations but with climatological sea ice and SST used in the Atmospheric Model Intercomparison Project (AMIP) as lower boundary conditions. The same resolutions are selected, that is, $96 \times 72,96 \times 96,144 \times 96,144 \times 144$, and $192 \times 144$. Each of these simulations last $10 \mathrm{yr}$.

Figure $1 \mathrm{~b}$ shows the distribution of the Southern Hemisphere jet positions in each of these atmosphereforced simulations and in the NCEP data. As in the coupled model, only an increase in latitudinal resolution of the atmospheric model is associated with a poleward shift and an increase in variance of the distribution: the mean position of the jet in the $96 \times 96$ $(144 \times 144)$ simulation is poleward of its mean position in the $96 \times 72(144 \times 96)$ simulation by $2.20^{\circ}\left(2.16^{\circ}\right)$. These shifts of the position are both significant at the $99 \%$ level $\left(p\right.$ value $\left.<2.2 \times 10^{-16}\right)$. The variance of the jet position in the $96 \times 96(144 \times 144)$ simulation is $1.72(1.61)$ times the one in the $96 \times 72(144 \times 96)$ simulation. These differences in variance are both significant at the $99 \%$ level ( $p$ value $=0.01$ and $p$ value $=0.003$, respectively).
While there is an improvement compared to the coupled model, even in the simulation with the highest resolution $(192 \times 144)$ the jet is still too equatorward by $2.63^{\circ} \mathrm{com}-$ pared to its position in the NCEP data, and the variance of its position is underestimated by a factor of 1.31 .

We now turn to idealized simulations, to explore a wider range of resolutions and to try to understand better the mechanisms responsible for the different responses to increases in the resolution in latitude or longitude.

\section{Dynamical core experiments}

\section{a. Idealized experiments: Design and validation}

To determine whether the impact of the horizontal resolution on the midlatitude atmospheric circulation may be due to the dynamical core of the LMDZ4 atmospheric model, we need to isolate the dynamics of the LMDZ4 from the physical parameterizations while preserving a realistic three-dimensional global circulation. Held and Suarez (1994) have proposed benchmark calculations satisfying these requirements to evaluate the dynamical cores of atmospheric general circulation models as a first step for intercomparison projects. Following their proposal, the detailed radiative, turbulence, and moist convective parameterizations are replaced with very simple forcing and dissipation. The forcing corresponds to a Newtonian relaxation of the temperature field to a zonally symmetric state, with an $e$-folding time scale of 40 days in the free troposphere increased to 4 days in the tropical boundary layer. The equilibrium temperature field is weakly stable on the vertical and becomes isothermal above $200 \mathrm{hPa}$. To mimic a Southern Hemisphere winter distribution, we add an $\varepsilon \sin ($ lat) term to the default equilibrium temperature profile given by Held and Suarez (1994), where $\varepsilon=10 \mathrm{~K}$. A Rayleigh damping of the low-level wind with an $e$-folding time scale of 1 day at the surface represents the boundary layer friction. There is no topography, in the sense that the surface is at constant geopotential. This method has already been used to investigate the sensitivity of the dynamical cores of different AGCMs to horizontal resolution: Canadian Climate Centre model, version 2 (CCC2) (Boer and Denis 1997), HadAM3 (Pope and Stratton 2002), GFDL (Gerber and Vallis 2007; Gerber et al. 2008), and ECHAM5 (Wan et al. 2008) and to vertical resolution (Williamson et al. 1998; Gerber et al. 2008). Still using the Held and Suarez (1994) design, Jablonowski (1998) and Ringler et al. (2000) validated their geodesic dynamical cores, Chen et al. (1997) compared the semi-Lagrangian and Eulerian schemes, and Chen et al. (2007) examined the role of surface friction on the midlatitudinal jet position. 
Before using the Held and Suarez (1994) proposal in our resolution sensitivity study, we first validate its application to the LMDZ4 atmospheric model in an experiment with a $96 \times 96$ resolution, which lasts $20 \mathrm{yr}$ of perpetual austral winter. The statistics computed on this simulation and shown on Fig. 2 can be compared with previous studies with other models (Held and Suarez 1994; Chen et al. 1997; Ringler et al. 2000; Wan et al. 2008). The butterfly-like structure in zonal wind simulated by the LMDZ4 is very similar to other models. A midlatitudinal westerly jet appears in each hemisphere near $50^{\circ}$ with a maximum wind around $250 \mathrm{hPa}$ of about $38 \mathrm{~m} \mathrm{~s}^{-1}\left(28 \mathrm{~m} \mathrm{~s}^{-1}\right)$ in the winter (summer) hemisphere. These jets close off with a well-defined reversed shear above $250 \mathrm{hPa}$. The surface westerlies reach about $15 \mathrm{~m} \mathrm{~s}^{-1}$ $\left(11 \mathrm{~m} \mathrm{~s}^{-1}\right)$ in the winter (summer) hemisphere. Subtropical jets can also be identified at about $30^{\circ}$ with a maximum wind of about $22 \mathrm{~m} \mathrm{~s}^{-1}\left(15 \mathrm{~m} \mathrm{~s}^{-1}\right)$ in the winter (summer) hemisphere. Easterlies appear in the equatorial and polar atmosphere. The zonal-mean temperature (Fig. 2b) corresponds to an austral winter distribution.

The eddy kinetic energy (Fig. 2c) and the eddy temperature variance (Fig. 2d) provide a measure of the amplitude of the baroclinic waves. ${ }^{1}$ A single maximum in kinetic energy appears in each hemisphere around $250 \mathrm{hPa}$ near $45^{\circ}$, that is, where the midlatitudinal jet resides. Two maxima in eddy temperature variance can be seen in each hemisphere: one in the lower troposphere, which extends upward and poleward, and another near the tropopause. The lower-troposphere maximum coincides with the maximum in poleward heat transport by the eddies (Fig. 2f). The eddies also transport momentum (Fig. 2e) poleward between $60^{\circ} \mathrm{S}$ and $60^{\circ} \mathrm{N}$ and equatorward between $60^{\circ}$ and $90^{\circ}$. The maxima in poleward momentum fluxes are located on the equatorward side of the jet in both hemispheres. Thus, the momentum fluxes converge at the location of the westerly jets in the midlatitudes, maintaining them against surface friction. The poleward heat fluxes (Fig. 2f) result in heat flux divergence in low latitudes and convergence in high latitudes. These features are in good agreement with those from previous studies.

We then perform a set of 10 additional idealized experiments with the same equilibrium temperature. The 11 simulations have different horizontal resolutions, dynamical time steps, and horizontal dissipation scales and each lasts $20 \mathrm{yr}$. The selected resolutions are $48 \times 48$,

\footnotetext{
${ }^{1}$ Eddies, defined as departures from the zonal average, normally include stationary and low-wavenumber barotropic waves. In the zonally symmetric setting used here, however, eddies are largely dominated by baroclinic waves and will be referred to as such.
}

$72 \times 48,96 \times 48,72 \times 72,96 \times 72,144 \times 72,96 \times 96$, $144 \times 96,96 \times 144,144 \times 144$, and $192 \times 144$. We tested the impact of the time step on the results presented below by running a simulation with a $48 \times 48$ resolution and with a time step of the $144 \times 144$ simulation. We did not find any significant impact of this change in time step on the midlatitudinal atmospheric circulation. We also tested the impact of the horizontal dissipation scale by running a simulation with a $72 \times 72$ resolution and the horizontal dissipation scale of the $144 \times 144$ simulation. We found that the impact of this change in horizontal dissipation scale is negligible relative to the impact of the combined change in horizontal resolution and horizontal dissipation scale.

\section{b. Characteristics of the jet in the idealized experiments}

Figure 3a shows the distribution of the Southern Hemisphere jet positions in each of the idealized experiments. As in the simulations with the full GCM, an increase in latitudinal resolution (color changes) at constant longitudinal resolution (same line style) is associated with a systematic poleward shift of the distribution by $2.25^{\circ}-11.24^{\circ}$. The midlatitude jet is also located poleward by $3.48^{\circ}$ in the $96 \times 96$ simulation compared to the $144 \times 72$ simulation. The total number of grid points is similar at these two resolutions, so they provide a good test of the impact of the anisotropy of the grid. These shifts of the midlatitudinal jet position are all significant at the $99 \%$ level. They are accompanied by poleward shifts of the midlatitude maximum in the zonal-mean meridional surface temperature gradient (Fig. 3b), except in the lowest-resolution case. The $48 \times 48$ simulation exhibits a curious behavior with a secondary midlatitude maximum around $50^{\circ} \mathrm{S}$, which is not consistent with the position of the midlatitudinal jet. The shifts of the midlatitudinal jet position when increasing the latitudinal resolution are also accompanied by an increase in variance of the jet position by a factor of 1.26-2.62, except when changing the resolution from $72 \times 48$ to $72 \times 72$ (Fig. 3a). The increases in variance are all significant at the $90 \%$ level. According to Gerber and Vallis (2007) and Gerber et al. (2008) results obtained applying Held and Suarez (1994) benchmark calculations, a poleward shift in the jet position is also accompanied by a decrease in the persistence of the northern annular mode. While a clear poleward shift and a clear increase in variance of the distribution are observed when increasing the latitudinal resolution, the changes are negligible when increasing the longitudinal resolution (change in line style).

To diagnose the impact of the horizontal resolution on the baroclinicity of the Southern Hemisphere jet, we 
96x96 idealized experiment: validation

a) Zonal-mean zonal wind $\left(\mathrm{m} \mathrm{s}^{-1}\right)$

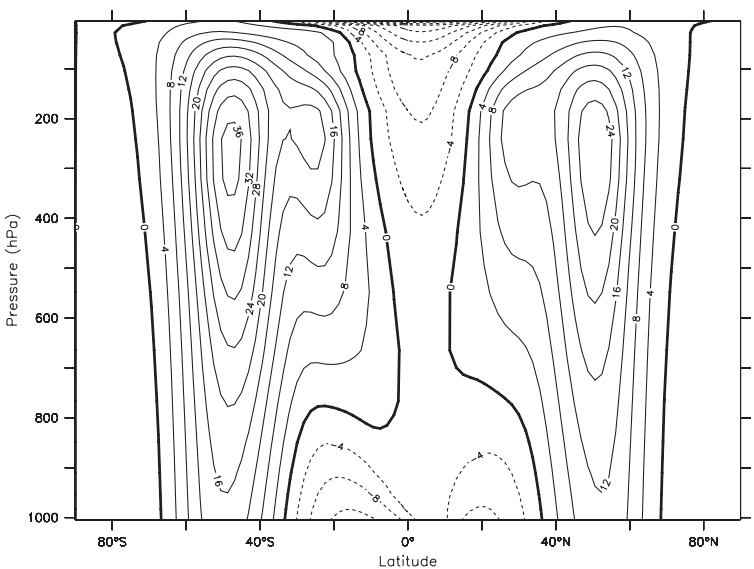

c) Eddy kinetic energy $\left(\mathrm{m}^{2} \mathrm{~s}^{-2}\right)$

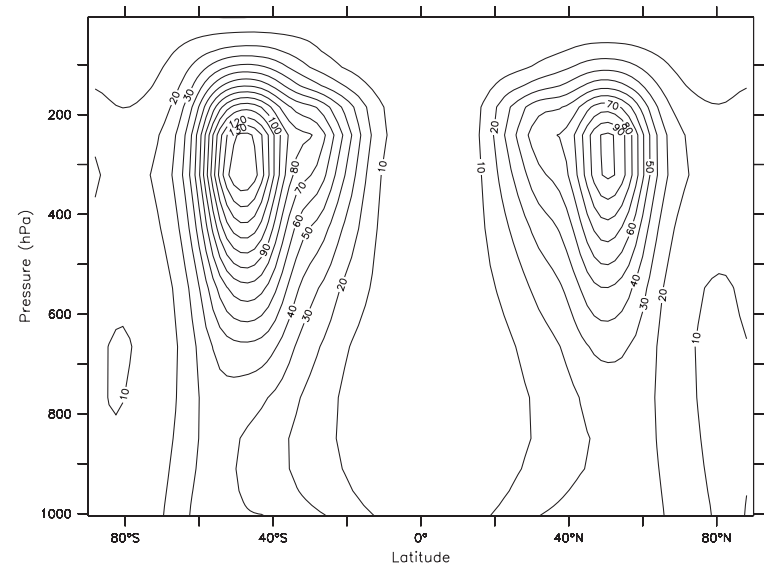

e) Eddy momentum flux $\left(\mathrm{m}^{2} \mathrm{~s}^{-2}\right)$

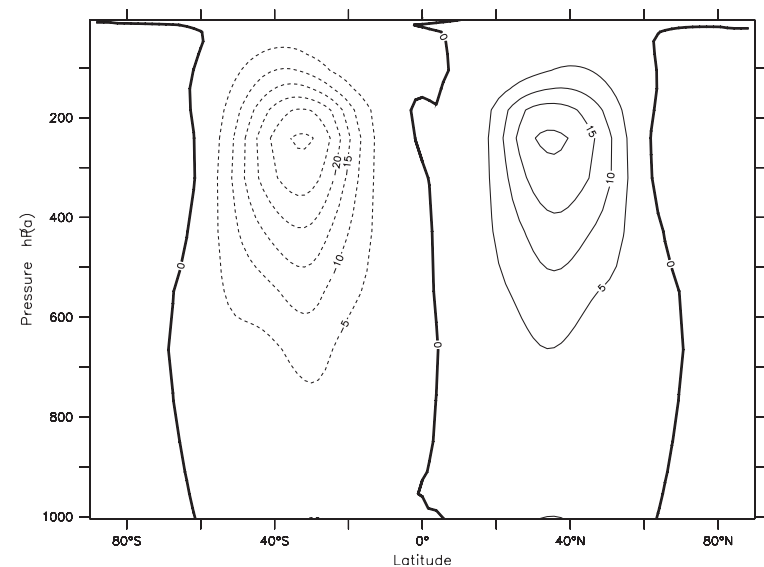

b) Zonal-mean temperature (K)

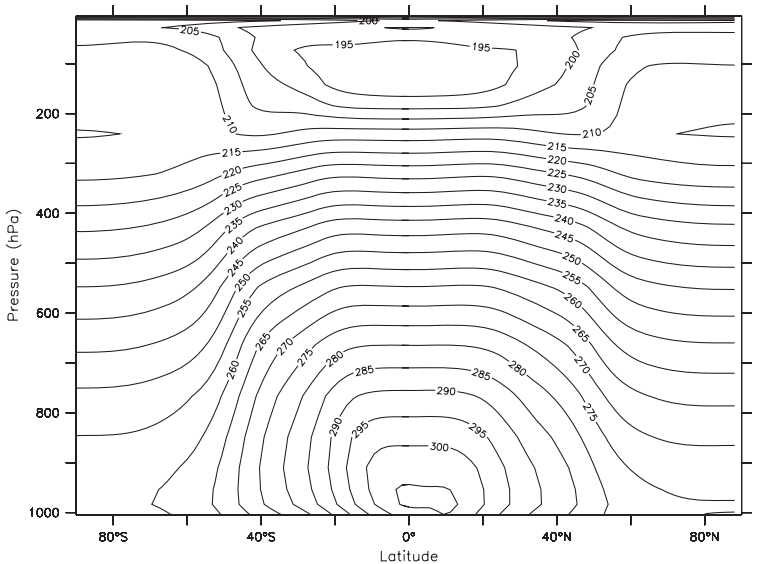

d) Eddy temperature variance $\left(\mathrm{K}^{2}\right)$

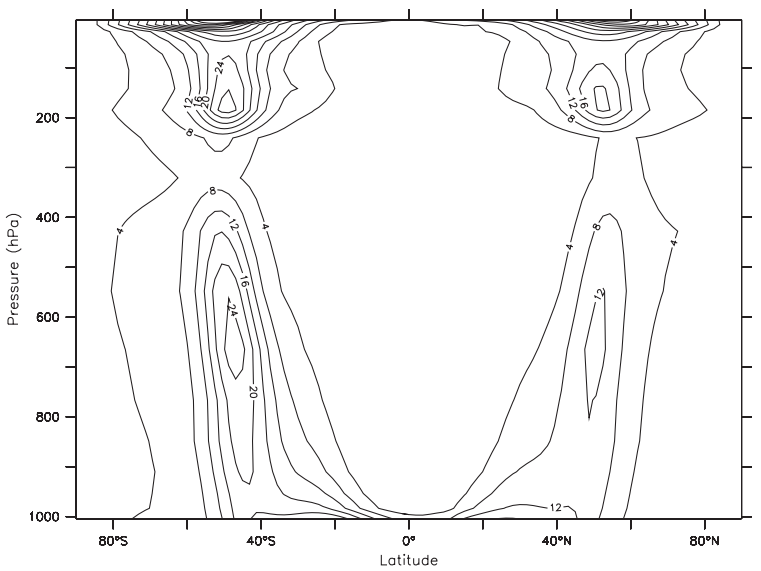

f) Eddy heat flux $\left(\mathrm{K} \mathrm{m} \mathrm{s}^{-1}\right)$

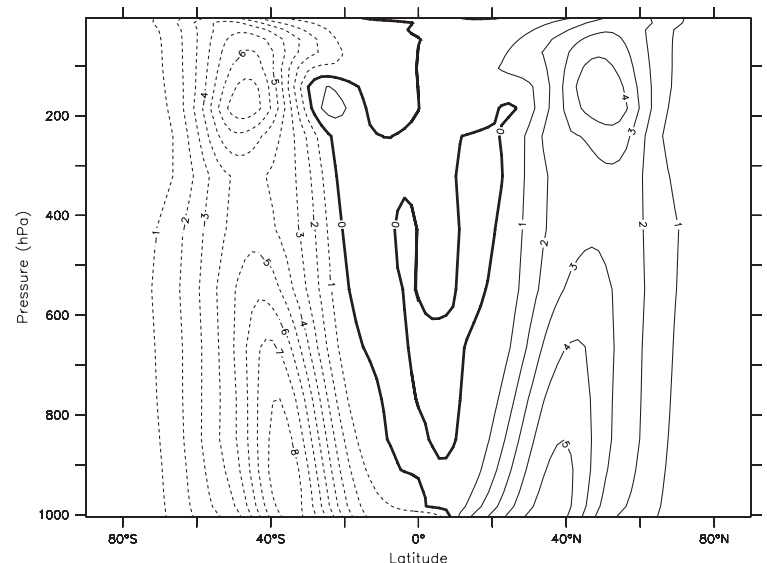

FIG. 2. The $96 \times 96$ idealized dynamical core experiment: zonal mean of (a) time-mean zonal wind, contour interval 4 m s ${ }^{-1}$; (b) timemean temperature, contour interval $5 \mathrm{~K}$; (c) eddy kinetic energy, contour interval $10 \mathrm{~m}^{2} \mathrm{~s}^{-2}$; (d) eddy temperature variance, contour interval $4 \mathrm{~K}^{2}$; (e) eddy momentum flux, contour interval $5 \mathrm{~m}^{2} \mathrm{~s}^{-2}$; and (f) eddy heat flux, contour interval $1 \mathrm{~K} \mathrm{~m} \mathrm{~s}^{-1}$. Continuous (dashed) contours indicate positive (negative) values. 
Free idealized experiments

a) Distribution of jet positions

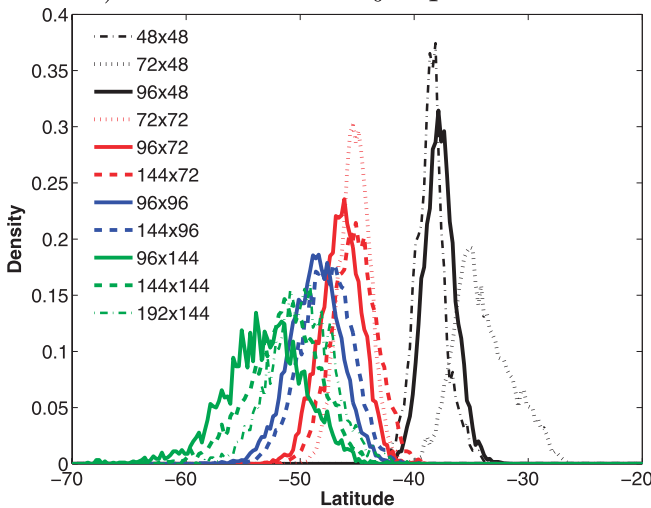

c) Baroclinicity at jet position
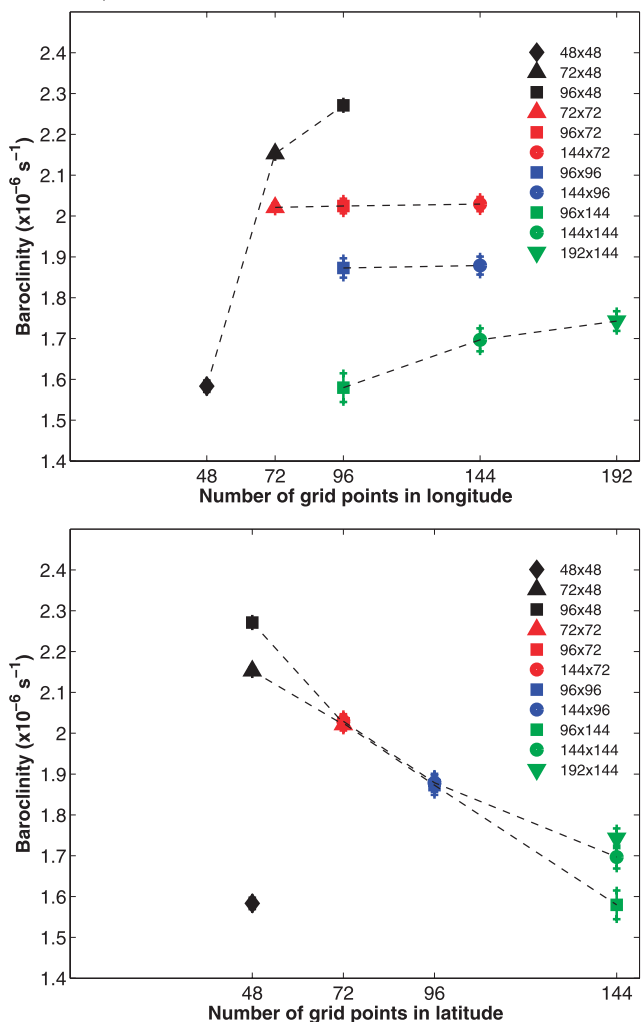

b) Surface meridional temperature gradient

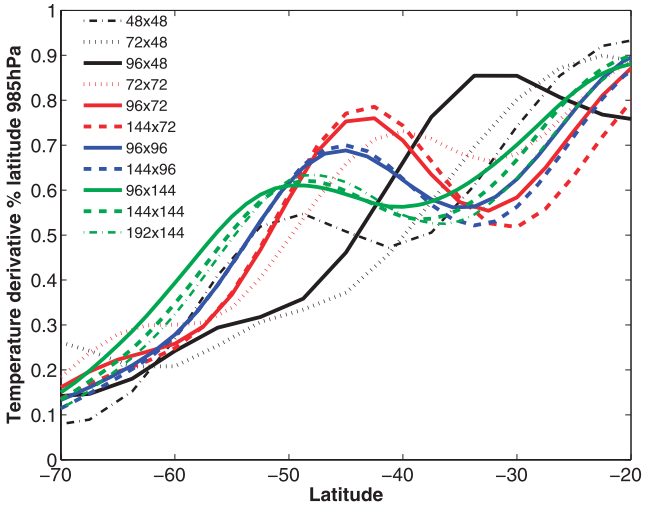

d) Ferrel cell strength
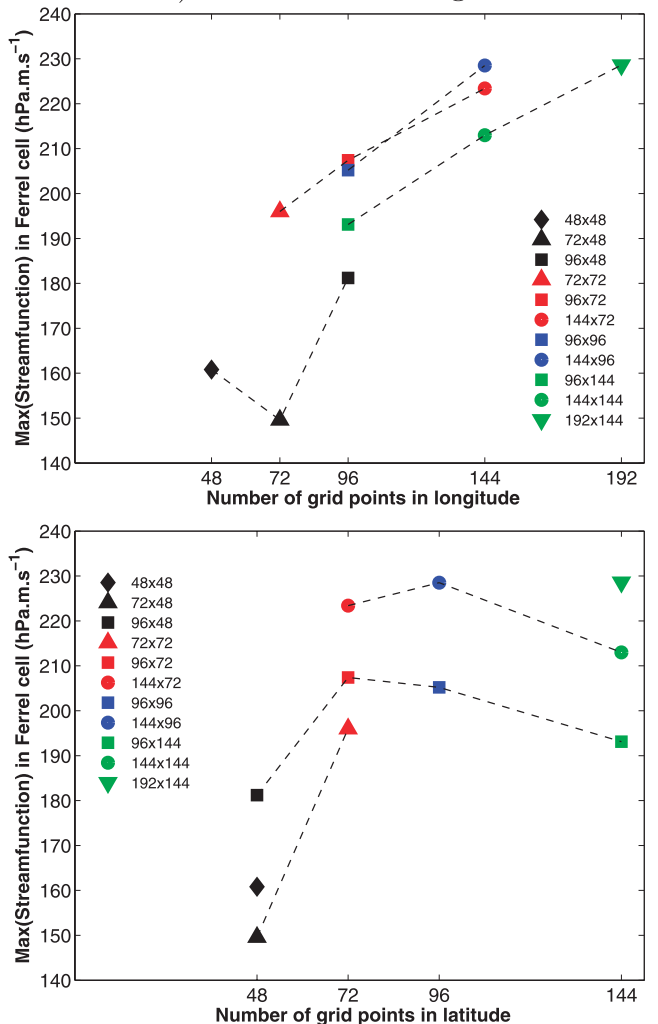

FIG. 3. Free idealized experiments: (a) PDF of the winter jet positions, computed as the latitude of the zonal-mean zonal wind maximum at the $850-\mathrm{hPa}$ level; (b) meridional temperature gradient at the $985-\mathrm{hPa}$ level, units $10^{-5} \mathrm{~K} \mathrm{~m}^{-1}$; (c) baroclinicity $\left(\mathrm{s}^{-1}\right)$, averaged between 200 and $1000 \mathrm{hPa}$ at the location of the jet; (d) strength of the Ferrel cell, units $\mathrm{hPa} \mathrm{m} \mathrm{s}$. In (c) and (d), the values are plotted as a function of the resolution in longitude (latitude), with dashed lines linking simulations with the same resolution in latitude (longitude) in the top (bottom) panels. The 95\% confidence interval is shown together with the mean value.

compute the mean baroclinicity between 200 and $1000 \mathrm{hPa}$ at the location of the jet. Figure $3 \mathrm{c}$ shows this mean baroclinicity as a function of the longitudinal (latitudinal) resolution in the top (bottom) panel with its
$95 \%$ confidence interval. When the latitudinal resolution increases at constant longitudinal resolution, the jet becomes more barotropic (significant at the $99 \%$ level using a $t$ test). The mean baroclinicity of the jet is also 
lower by $1.5 \times 10^{-7} \mathrm{~s}^{-1}$ in the $96 \times 96$ simulation than in the $144 \times 72$ simulation (significant at $99 \%$ ). This decrease of the baroclinicity occurs as the jet moves poleward, despite the increase in the absolute value of the Coriolis factor $f$, and is consistent with the lower value of the temperature gradient at the jet position (Fig. 3b). There is no systematic impact on the baroclinicity of the jet in these idealized simulations when changing the longitudinal resolution.

To complement these analyses, we diagnose the impact of the horizontal resolution on the strength of the Ferrel cell, computed as the maximum of the zonal-mean meridional streamfunction in the midlatitudes of the Southern Hemisphere. Figure 3d shows the strength of the Ferrel cell, defined in this way with its $95 \%$ confidence interval as a function of the longitudinal (latitudinal) resolution in the top (bottom) panel. The streamfunction maximum increases by $11.4-31.6 \mathrm{hPa} \mathrm{m} \mathrm{s}^{-1}$ when the longitudinal resolution increases at constant latitudinal resolution, except when changing the resolution from 48 $\times 48$ to $72 \times 48$. There is no systematic impact on the strength of the Ferrel cell in these idealized simulations when changing the latitudinal resolution. The streamfunction maximum is larger by $18.2 \mathrm{hPa} \mathrm{m} \mathrm{s}^{-1}$ in the $144 \times 72$ than in the $96 \times 96$ simulation.

In this section, we showed that in the LMDZ4 idealized experiments the following behavior is seen:

- When the latitudinal resolution increases, the Southern Hemisphere midlatitude jet moves poleward, together with the position of the midlatitude maximum of the temperature gradient. The variability of the jet position increases and the weaker temperature gradient leads to a reduced baroclinicity.

- When the longitudinal resolution increases, the Southern Hemisphere jet position, variability, and baroclinicity do not change but the Ferrel cell strengthens. This latter effect is not observed when the latitudinal resolution increases.

\section{c. Baroclinic wave amplitude}

In the midlatitudes, the atmospheric circulation is mainly driven by the baroclinic waves and the associated heat and momentum transports. To go farther, we thus need to assess the role of the horizontal resolution on the BWA, which we measure by the eddy kinetic energy at $240 \mathrm{hPa}$ (Fig. 4a) and by the eddy temperature variance at $850 \mathrm{hPa}$ (Fig. 4b). These levels are where the maximum variance is reached and are representative of the whole troposphere.

When the latitudinal resolution increases (color changes), the positions of both the maxima in eddy kinetic energy and in eddy temperature variance shift poleward: the sources of baroclinic waves follow the poleward shifts of the midlatitude jet and of the maximum temperature gradient. At the same time, the maxima in eddy kinetic energy and in eddy temperature variance decrease by 2.2 $52.8 \mathrm{~m}^{2} \mathrm{~s}^{-2}$ and $1.1-6.5 \mathrm{~K}^{2}$, respectively. This result can also be seen in Figs. 5a and 5b, which show the values of the maximum in eddy kinetic energy and eddy temperature variance between $20^{\circ}$ and $70^{\circ} \mathrm{S}$ as a function of the longitudinal (latitudinal) resolution in the top (bottom) panels for the same experiments as in Figs. $4 \mathrm{a}$ and $4 \mathrm{~b}$. This lower BWA with higher latitudinal resolution is consistent with the reduced baroclinicity.

On the contrary, when the longitudinal resolution increases (line style changes in Figs. $4 a$ and $4 b$ and symbol changes in Figs. 5a and 5b), the maxima in eddy kinetic energy and eddy temperature variance increase in magnitude by $29.5-102.6 \mathrm{~m}^{2} \mathrm{~s}^{-2}$ and $2.0-10.1 \mathrm{~K}^{2}$, respectively. This increase occurs with little change in the jet position or baroclinicity and can thus be seen as a consequence of the increased resolution. The stronger Ferrel cell with higher resolution in longitude may be driven by the higher BWA.

We can thus conclude that an increase in longitudinal resolution is associated with more energetic baroclinic waves, while an increase in latitudinal resolution is associated with less energetic ones. Most previous studies (Held and Suarez 1994; Boer and Denis 1997; Ringler et al. 2000; Pope and Stratton 2002; Wan et al. 2008) obtained an increase in BWA when increasing the horizontal resolution. Our results are consistent with theirs when the resolution is increased uniformly: both the maxima in eddy kinetic energy and eddy temperature variance increase when increasing the horizontal resolution from $48 \times 48$ to $72 \times 72$ and from $72 \times 72$ to $96 \times 96$, and the maximum in eddy kinetic energy also increases when increasing the horizontal resolution from $96 \times 96$ to $144 \times 144$. However, the separate analysis shows that this is because the increase in BWA brought by the increase in the resolution in longitude more than offsets the decrease in BWA when increasing the resolution in latitude.

\section{d. EP cross sections}

We now study the impacts of the changes of the wave characteristics on the mean flow using the EliassenPalm (EP) formalism. The meridional and vertical components of the EP fluxes are defined as follows:

$$
\begin{aligned}
& F_{y}=-\cos \varphi[u * v *] \text { and } \\
& F_{p}=f \cos \varphi \frac{\left[v * \theta^{*}\right]}{\partial_{P}[\theta]}
\end{aligned}
$$

where $\varphi$ is the latitude, [ ] denotes the zonal mean, asterisk denotes an anomaly relative to the zonal mean, $f$ is 
Free idealized experiments

a) 240-hPa eddy kinetic energy

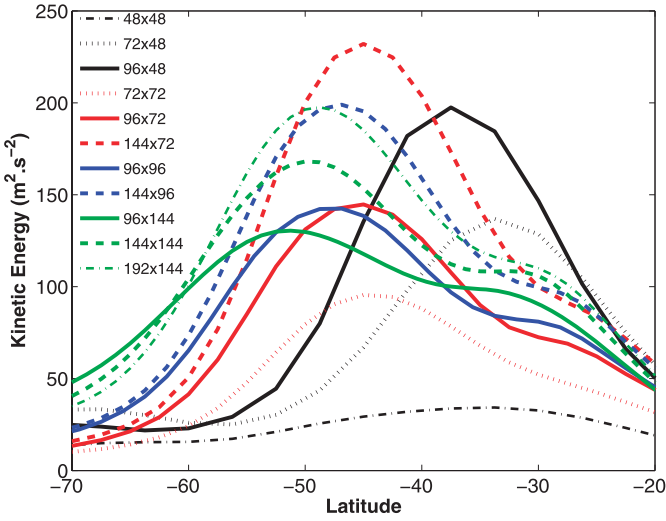

b) $850-\mathrm{hPa}$ eddy temperature variance

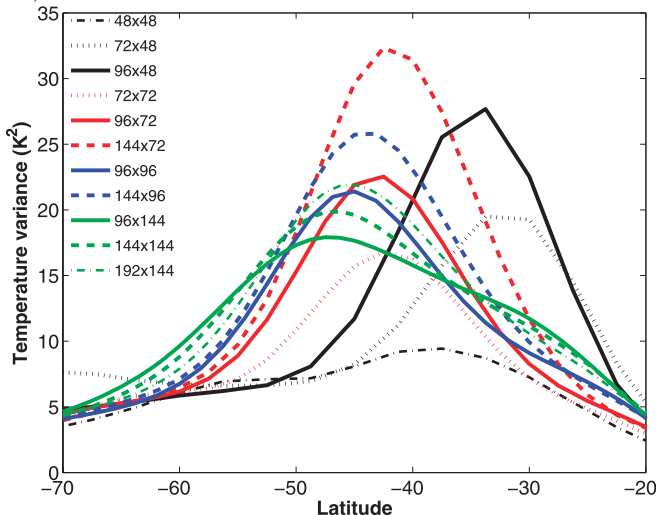

Constrained idealized experiments

c) 240-hPa eddy kinetic energy

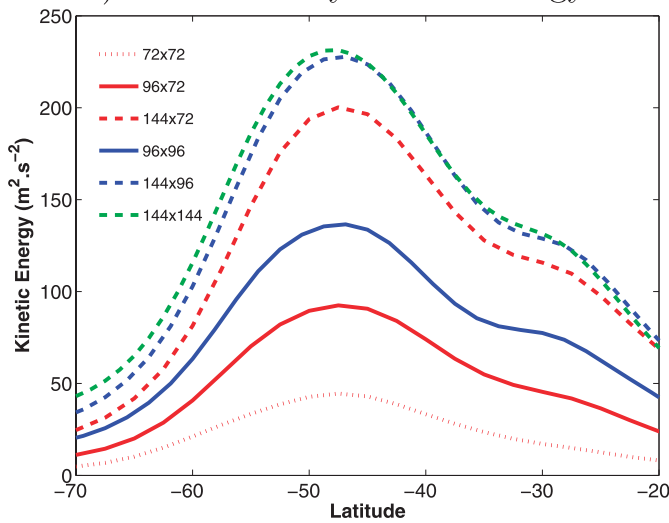

d) 850-hPa eddy temperature variance

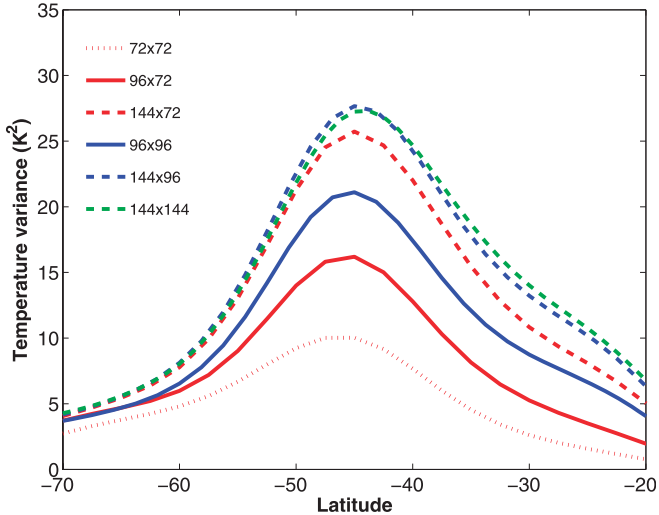

FIG. 4. Time and zonal averages for the idealized experiments: (top) the free experiments described in section 3a and (bottom) the constrained experiments described in section 4a for (a),(c) eddy kinetic energy, at the 240-hPa level $\left(\mathrm{m}^{2} \mathrm{~s}^{-2}\right)$, and (b),(d) eddy temperature variance at the $850-\mathrm{hPa}$ level $\left(\mathrm{K}^{2}\right)$.

the Coriolis parameter, $\theta$ is the potential temperature, $\partial_{P}$ corresponds to the partial derivative relative to pressure, and $u$ and $v$ are the zonal and meridional winds, respectively.

To illustrate the impact of the horizontal resolution on the generation and propagation of the baroclinic waves, we present meridional cross sections of Eliassen-Palm fluxes as a representative example of an increase in latitudinal resolution (Fig. 6, $96 \times 72$ to $96 \times 96$ ) and in longitudinal resolution (Fig. 6 b, $96 \times 96$ to $144 \times 96$ ). Both the differences (blue arrows) and the total field for the $96 \times 96$ simulation (black arrows) are shown. Note that the scale is 6 times larger for the black arrows than for the blue ones.

When increasing the resolution in latitude, the jet shifts poleward. The differences in EP fluxes then also show a shift in the low-level wave source, with an increase poleward of the $96 \times 96$ jet and a decrease equatorward. The loop is closed by enhanced equatorward propagation (poleward momentum transport) across the jet position. This EP flux configuration is reminiscent of the observed response to a shift of the jet through annular mode variability (Lorenz and Hartmann 2001) and leads to the maintenance of the jet at its new position.

When increasing the resolution in longitude, the characteristics of the jet remain very close, and we observe an increase in the EP fluxes in the low troposphere, which corresponds to an intensification of the baroclinic wave source. There is also a clear change in behavior of the waves on the equatorward flank of the jet, with an increased tendency toward poleward propagation (blue arrows directed to the left). The result is an equatorward momentum transport across the jet, inhibiting a poleward move. The larger heat fluxes and upper-level momentum 


\section{Free idealized experiments}

a) Max 240-hPa eddy kinetic energy
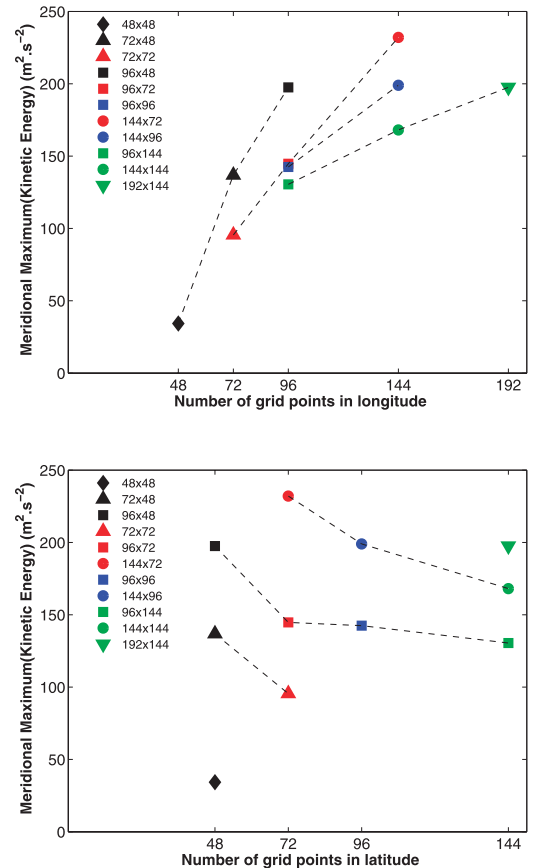

b) Max $850-\mathrm{hPa}$ eddy temperature variance
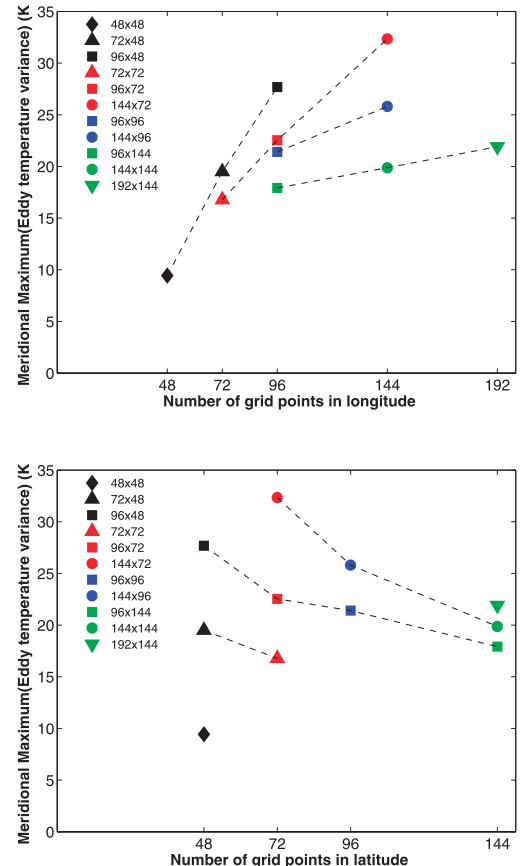

Constrained idealized experiments

c) Max 240-hPa eddy kinetic energy
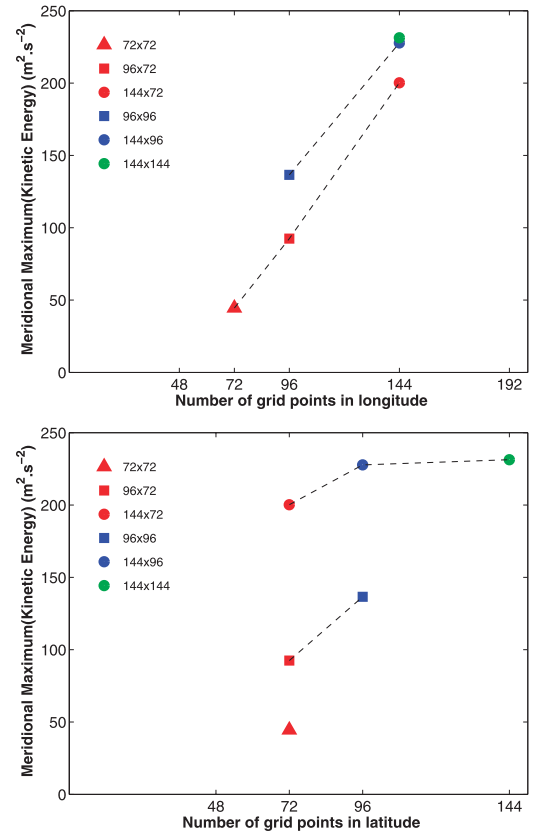

d) Max 850-hPa eddy temperature variance
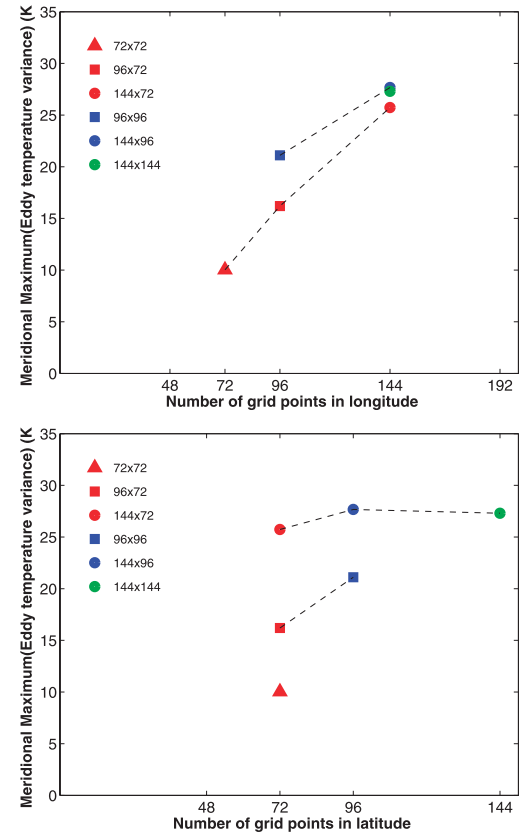

FIG. 5. Maximum of the time and zonal-mean of (left) eddy kinetic energy at $240 \mathrm{hPa}\left(\mathrm{m}^{2} \mathrm{~s}^{-2}\right)$ and (right) eddy temperature variance at $850 \mathrm{hPa}\left(\mathrm{K}^{2}\right)$ for (a),(b) free and (c),(d) constrained experiments. Maximum is taken between $20^{\circ}$ and $70^{\circ} \mathrm{S}$. Values are plotted as a function of the resolution in longitude (latitude), with dashed lines linking simulations with the same resolution in latitude (longitude) in top (bottom). 


\section{Free idealized experiments}

a) Eliassen-Palm fluxes (96x96-96x72)

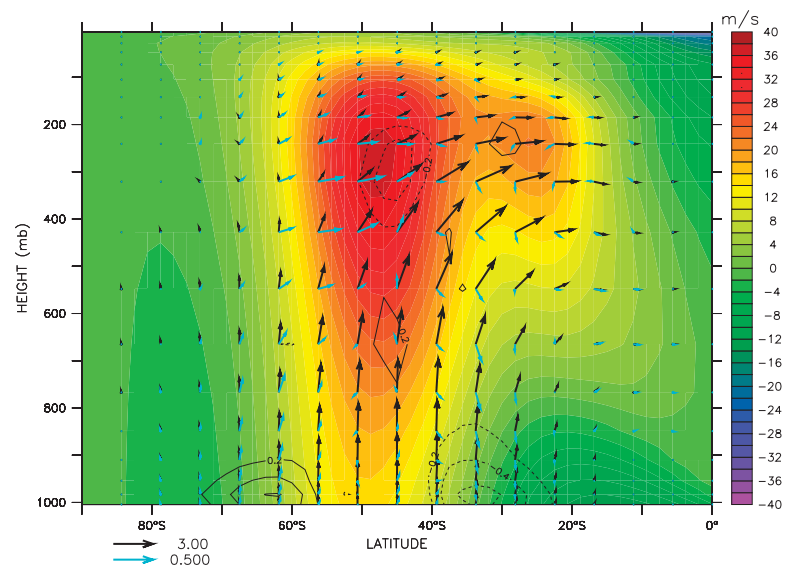

b) Eliassen-Palm fluxes (144x96-96x96)

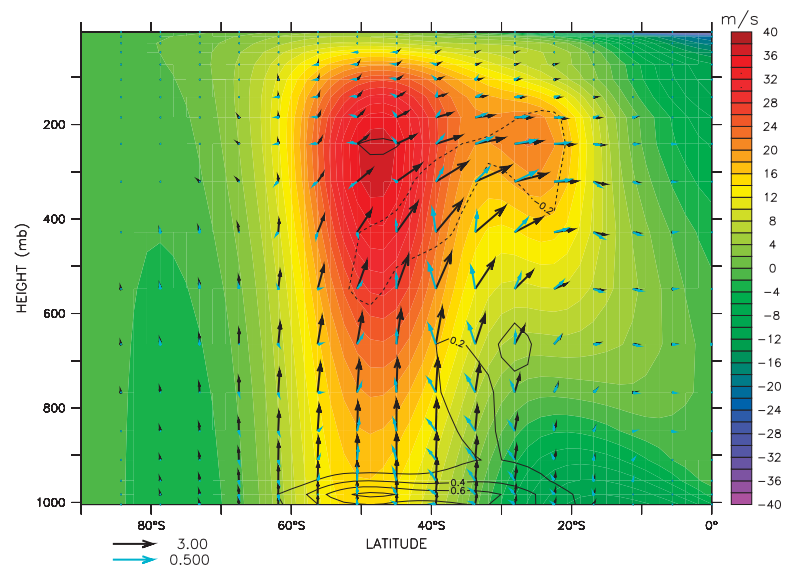

Constrained idealized experiments

c) Eliassen-Palm fluxes (96x96-96x72)

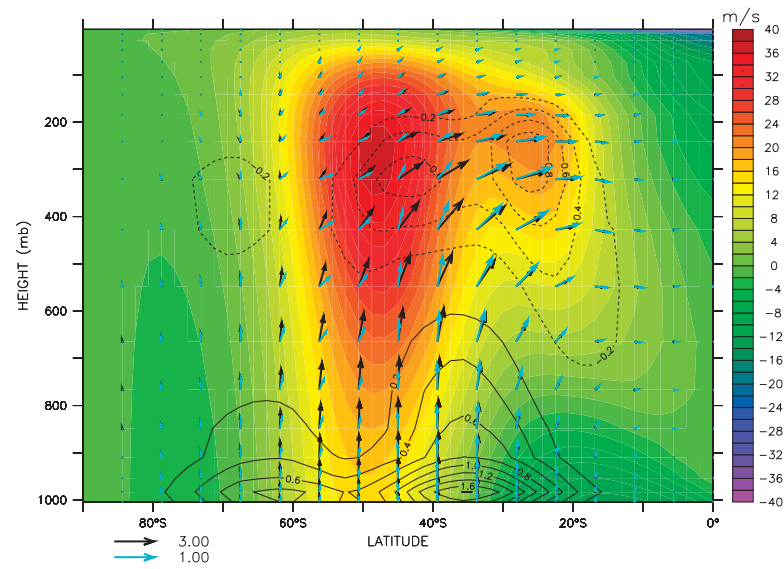

d) Eliassen-Palm fluxes (144x96-96x96)

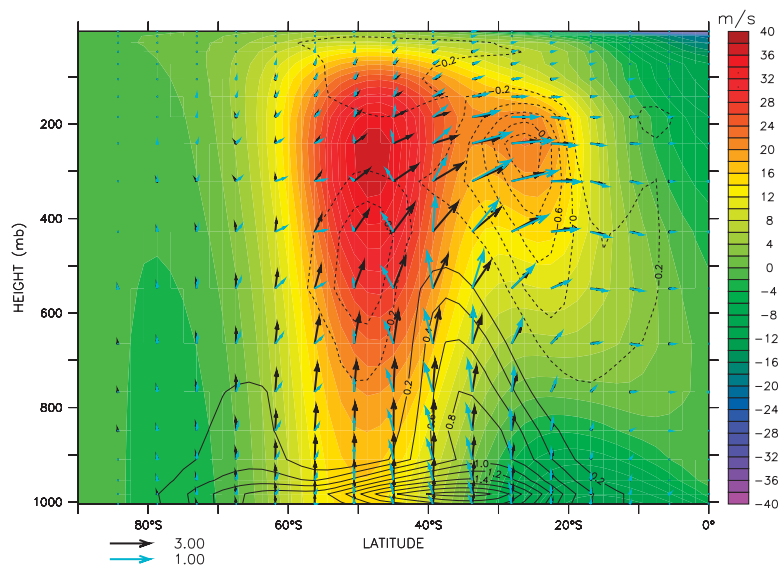

FIG. 6. Latitude-pressure cross sections of EP fluxes for selected (top) free and (bottom) constrained experiments. Color shading and black arrows: mean zonal wind and EP fluxes at the $96 \times 96$ reference resolution, interval $2 \mathrm{~m} \mathrm{~s}^{-1}$. Blue arrows and black contours: differences in EP fluxes and in their divergence between the simulations at the (a),(c) $96 \times 96$ and $96 \times 72$ and (b), (d) $144 \times 96$ and $96 \times 96$ resolutions. Contour interval: $0.2 \mathrm{~m} \mathrm{~s}^{-1}$ day $^{-1}$; dashed contours negative. Black arrow scale is 6 times the blue one in (a) and (b) and 3 times in (c) and (d).

flux convergence are compensated by the stronger Ferrel cell, which maintains the same wind shear and temperature gradient.

\section{e. Limitations}

In our set of idealized experiments, the midlatitudinal jet shifts poleward when increasing the resolution in latitude only. This is accompanied by a similar shift in the position of the maximal surface temperature, maintaining the thermal wind balance. The maximum of the eddy energy also follows the jet, but the reduced baroclinicity leads to less energetic waves. A last constraint is that the momentum transport by the eddies, mediated by the mean meridional circulation, must compensate the surface friction.

The observed changes in the waves and in the mean flow are thus consistent with each other, but it is very difficult to extract a cause that can be traced back to the changes in resolution: changes in the characteristics of the waves are probably largely forced by changes in the 
TABLE 1. Maximum in the Southern Hemisphere troposphere of the absolute differences in time mean: EKE ( $\left.\mathrm{m}^{2} \mathrm{~s}^{-2}\right)$, eddy temperature variance $\left(\mathrm{K}^{2}\right)$, eddy momentum flux $\left(\mathrm{m}^{2} \mathrm{~s}^{-2}\right)$, and eddy heat flux $\left(\mathrm{K} \mathrm{m} \mathrm{s}^{-2}\right)$ between the $96 \times 96$ constrained experiment and the $96 \times 96$ free experiment, between the halves of the $96 \times 96$ free experiment, between the $144 \times 96$ and the $96 \times 96$ free experiments, and between the $96 \times 96$ and the $96 \times 72$ free experiments.

\begin{tabular}{lcccc}
\hline \hline \multicolumn{4}{c}{ Validation of the $96 \times 96$ constrained experiment } & \\
\hline \multicolumn{1}{c}{ Expts } & EKE & {$\left[T^{* 2}\right]$} & {$\left[U^{*} V^{*}\right]$} & $\mathrm{K}^{2}$ \\
$\mathrm{~m}^{2} \mathrm{~s}^{-2}$ & 2.0 & $\left.\mathrm{~K}^{*} \theta^{*}\right]$ \\
\hline $96 \times 96$ constrained-96 $\times 96$ free & $\mathrm{m}^{2} \mathrm{~s}^{-2}$ & 0.8 & 0.7 & 0.7 \\
Half $196 \times 96$ free-half $296 \times 96$ free & 6.6 & 0.4 & 13.6 & 0.3 \\
$144 \times 96$ free-96 996 free & 4.4 & 7.8 & 12.0 & 1.1 \\
$96 \times 96$ free-96 $\times 72$ free & 63.1 & 6.2 & 1.0 \\
\hline
\end{tabular}

mean flow, and the waves in turn force these mean flow changes.

To further understand the role of resolution changes, we need to cut this eddy-mean flow coupling. To that end, we will run a series of experiments with varying resolution in which the zonal-mean zonal wind is held at the same value. The forcing of the mean flow by the eddies is then removed, enabling the isolation of the direct response of the waves to resolution changes. The design and results of these "constrained" experiments are presented below.

\section{Constrained experiments}

\section{a. Constrained experiments: Design and validation}

The $96 \times 96$ idealized experiment defined in the previous section was chosen as a reference state for another set of simulations with different resolutions. In these constrained simulations, the zonal-mean zonal wind is restored toward its time-varying counterpart from the reference simulation with a time scale of one day, by adding a term in the zonal momentum equation:

$$
\frac{\partial[u]}{\partial t}=-\frac{1}{\tau}\left([u]-\left[u_{\text {ref }}\right]\right) .
$$

This method, and the restoring time scale $\tau$, was chosen over other ones with an additional relaxation of the zonal-mean temperature or meridional wind, or with relaxation toward the long-term mean. To pick the best method, we ran sensitivity experiments with relaxation at the same $96 \times 96$ resolution, and we validated the strength of the baroclinic wave sources, their directions of propagation, the associated heat and momentum transports, and their temporal variability against the reference free $96 \times 96$ experiment. We also checked that the zonal-mean zonal wind remained very close to the one from the reference experiment when changing the resolution.
Table 1 shows the differences in time-mean eddy kinetic energy, temperature variance, momentum, and heat fluxes between the $96 \times 96$ constrained and free (reference) experiments. The same differences between the halves of the $96 \times 96$ free experiment provide a measure of the expected noise for comparison. The values shown are the maxima of the absolute differences in the Southern Hemisphere troposphere. For each of the mentioned eddy statistics, the $96 \times 96$ constrained experiment is slightly farther from the $96 \times 96$ free experiment than the weather noise. The same eddy statistics are also computed in the free experiments for changes in resolution from $96 \times 96$ to $144 \times 96$ or $96 \times$ 72 . The maximum in absolute kinetic energy differences is 5-10 times larger between these couples of free experiments than between the $96 \times 96$ constrained and free ones. The difference in temperature variance is 8 10 times larger and 6-7 times larger for momentum fluxes. Thus, the error induced by the constraining method is small compared to the differences in the baroclinic wave characteristics that we aim at explaining. Only in the eddy heat flux case is the maximum in absolute differences between the $96 \times 96$ constrained and free experiments similar to the one between the couples of free experiments. This maximum error is located at the lowest level of the troposphere in the midlatitudes and corresponds to a weakened source of baroclinic waves in the constrained experiment.

The method was used for six experiments at the resolutions of $72 \times 72,96 \times 72,96 \times 96,144 \times 72,144 \times 96$, and $144 \times 144$. All were restored to the $96 \times 96$ reference state. To test the robustness of the results presented below, we also ran additional experiments at the resolutions of $96 \times 72,96 \times 96$, and $144 \times 96$ but in which the reference state was changed to the zonalmean state of the $96 \times 72$ free experiment, in which the jet is located equatorward. The results from this second set of sensitivity experiments agree well with the ones from the first set, so we will only present the results from the first set in the following. 
As a result of the strong relaxation, the zonal-mean zonal wind is almost identical in all the constrained experiments. Any impact of changes of the eddies or mean meridional circulation with the resolution is canceled by the restoring term of Eq. (2). The zonal-mean meridional and vertical circulations are not constrained, and adjust so that the zonal-mean temperature gradient also remains close to the reference simulation, thereby maintaining the thermal wind balance. Thus, we do not show changes in the jet structure, temperature gradient, or baroclinicity with resolution for the constrained experiments as they are all very small.

\section{b. Baroclinic wave amplitude}

The 240-hPa eddy kinetic energy and 850-hPa eddy temperature variance in the constrained experiments are shown on Fig. 4. We can first note that the results are the same in the $96 \times 96$ free and constrained experiments, validating the method. As the positions of the jet and the maximum temperature gradient do not change in the constrained experiments, the maxima of BWA do not move either. As shown in Figs. 5c and 5d, the values of the maxima both increase with the longitudinal resolution; however, while they decrease in the free experiments when the latitudinal resolution increases, they also increase in the constrained experiments. This suggests that the decrease in amplitude observed in the free experiments was due to the shift of the jet and reduced baroclinicity, and not directly to the change in resolution. The energy of the waves eventually converges above the $144 \times 144$ resolution.

\section{c. EP cross sections}

To illustrate the changes of behavior of the waves and their potential influence on the zonal-mean flow, we now present meridional cross sections of the differences of the Eliassen-Palm fluxes (Fig. 6) for the same changes of resolution as shown for the free experiments. We will show in the next subsections that these examples are representative of the behavior of the complete set. Both the differences (blue arrows) and the total field for the $96 \times 96$ simulation (black arrows) are shown. Note that the scale is 3 times larger for the black arrows.

When increasing either the latitudinal or longitudinal resolution, we obtain a large increase in the EP fluxes in the low troposphere, which corresponds to an intensification of the baroclinic wave sources. For the increase in latitude resolution (Fig. 6c), the differences in EP fluxes look like an amplification of the total fluxes: the additional waves propagate mostly equatorward in the same way as in the reference simulation, increasing the poleward momentum transport across the jet. If the relaxation constraint is removed, the jet moves poleward as a result of the increased momentum transport, as it does in the free experiments. The EP fluxes are then influenced by changes in the zonal-mean wind and temperature fields, and the equilibrium response (Fig. 6a) is very different from the direct response to the resolution change seen in the constrained experiment.

When the resolution increase is in longitude (Fig. 6d), there is instead a clear increase in poleward propagation on the equatorward flank of the jet, between $40^{\circ}$ and $45^{\circ}$ south (blue arrows directed left). This gives a weaker poleward momentum transport across the jet with the higher resolution, preventing a jet shift. The EP flux differences in this situation are qualitatively similar to the ones between the corresponding free experiments (Fig. 6b), but the intensification of the wave source is much larger in the constrained experiment. We will see later that this larger wave source is not sustainable (section 4e).

\section{d. Generalization to the whole set of experiments}

We now check whether the two particular examples discussed above are really representative of all increases in resolution. As we will see, the EP flux changes when increasing the resolution in latitude or in longitude indeed behave as in the corresponding example. The exception is the increase in longitude from $72 \times 72$ to $96 \times$ 72 points, which has intermediate characteristics, both in the free and constrained experiments. It is also the only case in which the jet moves slightly poleward (instead of equatorward) with increased longitude resolution. The results for this particular change of resolution are shown for completeness but are not specifically commented on here.

Figure 7a shows the difference in the vertical component of the Eliassen-Palm flux at the 550-hPa level between couples of constrained experiments with varying resolution in latitude (red curves) or in longitude (blue curves). The position of the jet is noted by a dashed line. There is a systematic increase of wave activity entering the upper troposphere when increasing the horizontal resolution, strongest equatorward of the jet, and with an increase in longitudinal resolution.

In the free experiments (Fig. 7c), the increase of upward wave activity flux with increased longitude resolution has a similar profile as the constrained experiments but with a much reduced amplitude: the heat flux increase in the constrained experiment is not sustainable (see section $4 d$ ). When increasing the resolution in latitude, the wave sources do not increase as in the constrained experiments but shift poleward with the jet, and the differences (in red) consist of a dipole with a decrease (increase) in wave activity equatorward (poleward) of the jet. 


\section{Constrained idealized experiments}

a) Vertical component of EP flux $550 \mathrm{hPa}$

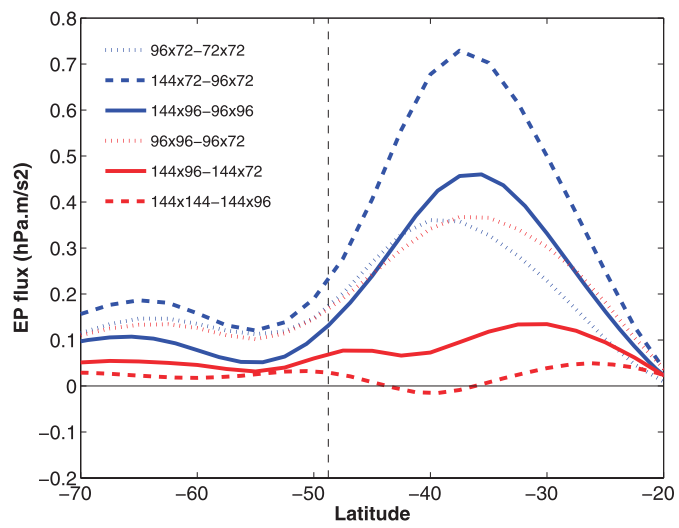

b) Horizontal component of EP flux (100-1000 hPa)

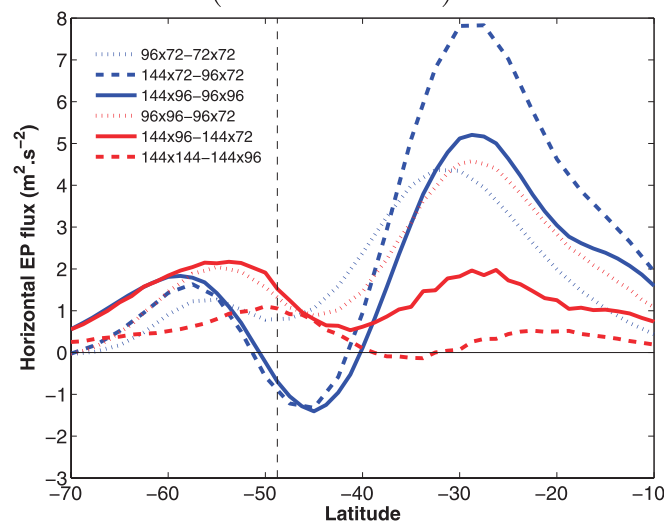

Free idealized experiments

c) Vertical component of EP flux $550 \mathrm{hPa}$

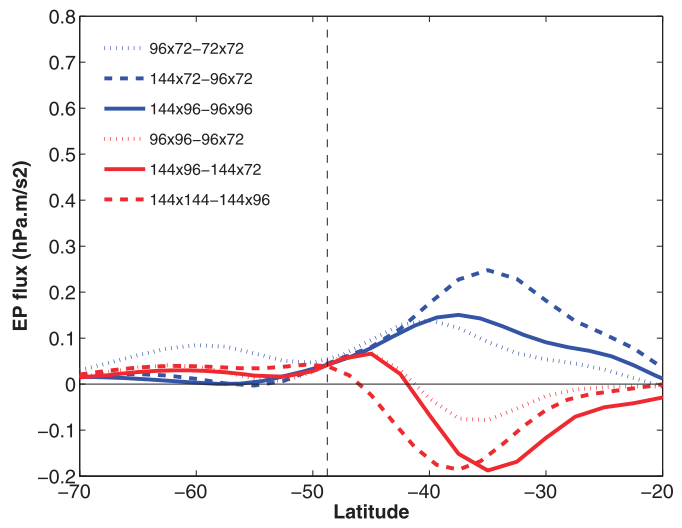

d) Horizontal component of EP flux (100-1000 hPa)

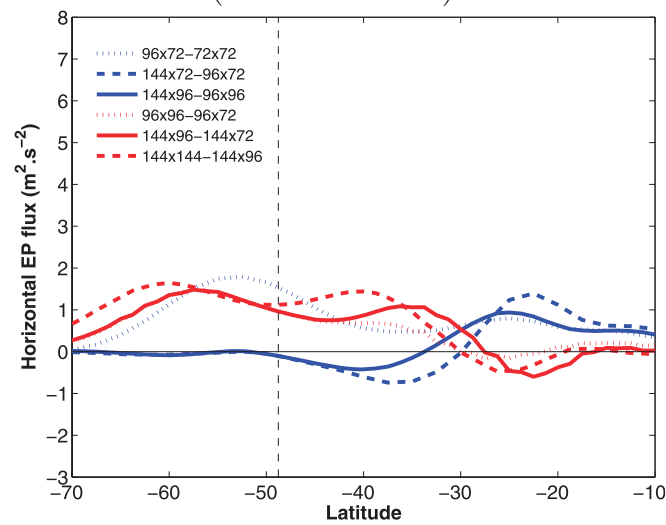

FIG. 7. Components of the EP fluxes as a function of latitude for the (top) constrained and (bottom) free experiments. Differences between the pairs of simulations are shown, with an increase of the resolution either in latitude (red lines) or in longitude (blue lines); the resolution changes and corresponding line styles are identical in each panel: (a),(c) vertical component at the 550-hPa level $\left(\mathrm{hPa} \mathrm{m} \mathrm{s}^{-2}\right.$ ) and (b),(d) horizontal component averaged between 100 and $1000 \mathrm{hPa}\left(\mathrm{m}^{2} \mathrm{~s}^{-2}\right)$. Vertical black dashed line gives the position of the midlatitude jet in the constrained experiments.

The changes in the total poleward momentum transport by eddies in the troposphere, given by the horizontal component of the Eliassen-Palm flux integrated from 100 to $1000 \mathrm{hPa}$, are shown in Fig. $7 \mathrm{~b}$ for the constrained experiments. When the horizontal resolution increases, there is in all cases an increase in poleward momentum transport peaking in the subtropics. It appears to be a direct consequence of the increased wave source immediately poleward, and their amplitudes are indeed related. Another relative maximum occurs poleward of the jet, between 50 and $60^{\circ} \mathrm{S}$. The most important difference between increases in the resolution in latitude or in longitude occurs close to the jet position: with an increase in latitude there remains an increased poleward momentum flux across the jet, while the momentum flux difference becomes equatorwardor very small-for an increase in longitude. The large additional wave activity that develops equatorward of the jet propagates partly poleward, preventing a shift of the jet. 
In the free experiments (Fig. 7d), the momentum flux changes with increased resolution in longitude again look like a lower-amplitude version of their constrained counterpart, with a reduced poleward flux just equatorward of the jet position. The picture is different for an increase in latitude resolution: there is then a broad increase in poleward momentum transport throughout the midlatitudes, consistent with the changes in wave sources.

The different behaviors seen on the EP flux cross sections (Fig. 6) thus hold for other increases of the resolution in latitude or longitude. The upward EP flux always increases with resolution in the constrained experiments; however, the resulting changes in momentum flux across the jet are of opposite signs when the resolution increase is in latitude or in longitude, explaining the different movements of the jet. The changes in the waves with an increase in latitude resolution in the free experiments are quite different from the direct response with a constrained jet; they can thus be understood as a coupled wave-mean flow response to the jet shift.

\section{e. A self-maintaining jet?}

In the constrained experiments, in which the zonalmean zonal wind follows the one at the $96 \times 96$ resolution, the low-level heat flux by the waves increases with resolution on the equatorward side of the jet, especially with an increase in longitude. When the waves propagate away from their source region, this in turn leads to a strong momentum flux convergence around $35^{\circ} \mathrm{S}$ (Fig. 7b), which would accelerate the flow. However, no corresponding change in the zonal-mean wind is observed in the equivalent unconstrained experiments at this latitude, which is located between the subtropical and midlatitude jets (Fig. 3). The changes in EP fluxes are indeed much weaker in the free experiments (Fig. 7d).

An increase in the poleward heat flux would normally tend to destroy the low-level baroclinicity and therefore remove the wave source in a negative feedback loop. In the constrained experiments, the zonal-mean meridional circulation, which is free to evolve, adjusts to changes in the heat flux to maintain a temperature gradient in balance with the imposed zonal wind. In a free atmosphere, the conditions for such a self-maintaining jet have been described by Robinson (2006), whose theory is illustrated in Fig. 8a. The crucial condition for a self-maintaining jet is for the divergence of the EP fluxes in the upper troposphere to have two minima on each side of the jet and a relative maximum centered on the midlatitudinal jet (black curve). In this case, the net effect of baroclinic waves in the upper troposphere is to slow down the zonal wind (in purple) to a larger extent on the jet wings than in the jet core. A secondary circulation then appears, balancing these effects through the Coriolis force. The meridional wind is larger on each side of the jet than at its center (in green), giving by continuity an ascent (descent) on the pole side (equator side) of the jet, causing a cooling (warming) in the low troposphere (in red). The low-level baroclinicity is then reinforced right at the jet position, helping to maintain a wave source there.

Figure $8 \mathrm{~b}$ shows the total divergence of the EliassenPalm fluxes averaged over the 200-500-hPa layer, representing the upper troposphere, for each of the six constrained experiments. Two points can be highlighted in this figure. First, when the latitudinal resolution increases, the relative maximum in divergence shifts poleward. It is about $1^{\circ}-6^{\circ}$ poleward in the simulations with 96 (blue) or 144 (green) latitudinal grid points compared to the simulations with 72 (red) points in latitude. Second, in all of the constrained experiments, a strong minimum is observed on the equator side of the jet. Thus, a poleward shift in the midlatitudinal jet position when increasing the latitudinal resolution would be self-maintaining, while an equatorward shift of the jet and an acceleration around $35^{\circ} \mathrm{S}$ would not, according to Robinson (2006)'s criteria. Indeed, the increase in low-level heat fluxes around $35^{\circ} \mathrm{S}$ is less strong in free experiments. The increase in upper-level total EP flux divergence on the pole side of the jet but not on the equator side of the jet when increasing either the latitudinal or the longitudinal resolution can also be observed on the EP cross sections (Fig. 6).

\section{Discussion}

\section{a. On the changes in wave behavior}

The increase in baroclinic wave amplitude when increasing the horizontal resolution in the idealized experiments has been observed in other models: Wan et al. (2008) obtained the same result in a similar Held and Suarez (1994) framework. They suggested that this increase could be explained by the fact that a higher horizontal resolution is able to resolve motions at smaller scales and allows for more energetic waves because of the growth of instabilities.

However, this does not explain the change in the direction of propagation of the waves when increasing the resolution in longitude. A possibility would be that a higher resolution in longitude allows to better resolve higher zonal wavenumbers, which tend to propagate preferentially poleward and break cyclonically. To test this hypothesis, we performed a spectral analysis on the eddy heat and momentum fluxes at each tropospheric 
a) Schematic of Robinson (2006) theory

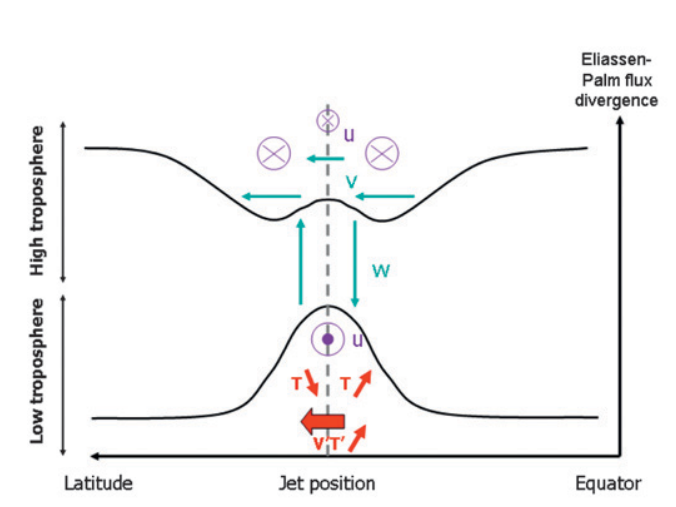

b) Total divergence of EP flux (200-500 hPa)

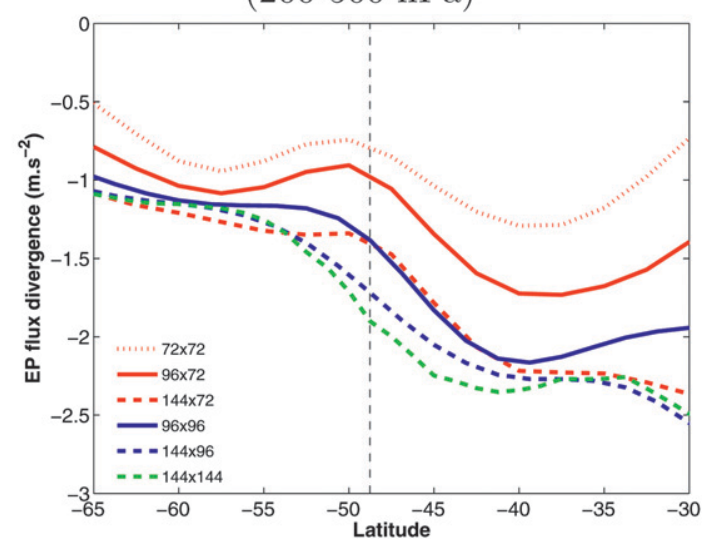

FIG. 8. (a) Schematic describing the role of secondary circulations induced by baroclinic waves in the selfmaintenance of midlatitudinal jets according to the theory of Robinson (2006). (b) Time-mean total divergence of the EP fluxes averaged over the 200-500-hPa layer in the constrained experiments. Units: $\mathrm{m} \mathrm{s}^{-1}$ day $^{-1}$. Vertical black dashed line gives the position of the midlatitudinal jet.

level in the constrained experiments (not shown). Results showed that the increase in BWA is always strongest over the dominant wavenumbers 5-7 and do not exhibit a preference toward higher wavenumbers with an increase in longitudinal resolution.

Orlanski (2003) obtained a bifurcation from predominant anticyclonic to predominant cyclonic wavebreaking in their model when the near-surface baroclinic development intensifies, as is observed with an increase of resolution. The increase of the wave sources when increasing the resolution in longitude is also very strong equatorward of the jet (Fig. 7), perhaps because the zonal gridpoint size is higher there. These waves then tend to propagate poleward more than waves originating from higher latitudes.

These possible mechanisms do not, however, explain why there is no poleward propagation with an increase in the resolution in latitude: wave sources also increase in that case, even if a bit less, and the result is poleward momentum flux. It is possible that the finer resolution in latitude favors equatorward propagation and anticyclonic wavebreaking by allowing high meridional wavenumbers, as proposed by Held and Phillipps (1993).

\section{b. Comparison with the full GCM}

We can wonder whether the impacts of the horizontal resolution on the midlatitude circulation diagnosed in the idealized experiments carry over to the full $\mathrm{LMDz}$ GCM. The results presented in sections $2 b$ and $2 c$ showed that it does for the changes in the position of the jet.

\section{1) BAROCLINICITY}

Figure 9a shows the mean baroclinicity between 200 and $1000 \mathrm{hPa}$ at the location of the jet computed on the five coupled experiments described in section $2 b$ as a function of the longitudinal (latitudinal) resolution in the top (bottom) panel, with its $95 \%$ confidence interval. When the latitudinal resolution increases, the jet becomes more barotropic, which follows the behavior highlighted in the free idealized experiments. On the contrary, when the longitudinal resolution increases, the jet remains at its position in latitude but becomes more baroclinic, a behavior that was not observed in the idealized experiments. These changes in baroclinicity of the jet when increasing the horizontal resolution are mainly due to changes in the upper-troposphere zonal wind (not shown) and are significant at the $99 \%$ level.

\section{2) FERREL CELL}

Figure $9 \mathrm{~b}$ shows that the Ferrel cell seems to strengthen when the horizontal resolution increases in the IPSL CM4 coupled model either in latitude or in longitude, if we except the $96 \times 96$ to $144 \times 96$ case. The streamfunction maximum is larger by $9.1,13.6$, and $20.9 \mathrm{hPa} \mathrm{m} \mathrm{s}$ in the $192 \times 144$ than in the $144 \times 144$ simulation, in the $144 \times$ 96 than in the $96 \times 72$ simulation and in the $96 \times 96$ than in the $96 \times 72$ simulation, respectively. These differences are significant at the $90 \%$ level (first one) or $99 \%$ level (last two). The strengthening of the Ferrel cell when increasing the longitudinal resolution is consistent with the results obtained in the free idealized experiments. On the contrary, when the latitudinal 
Coupled simulations

a) Relation between baroclinicity and resolution
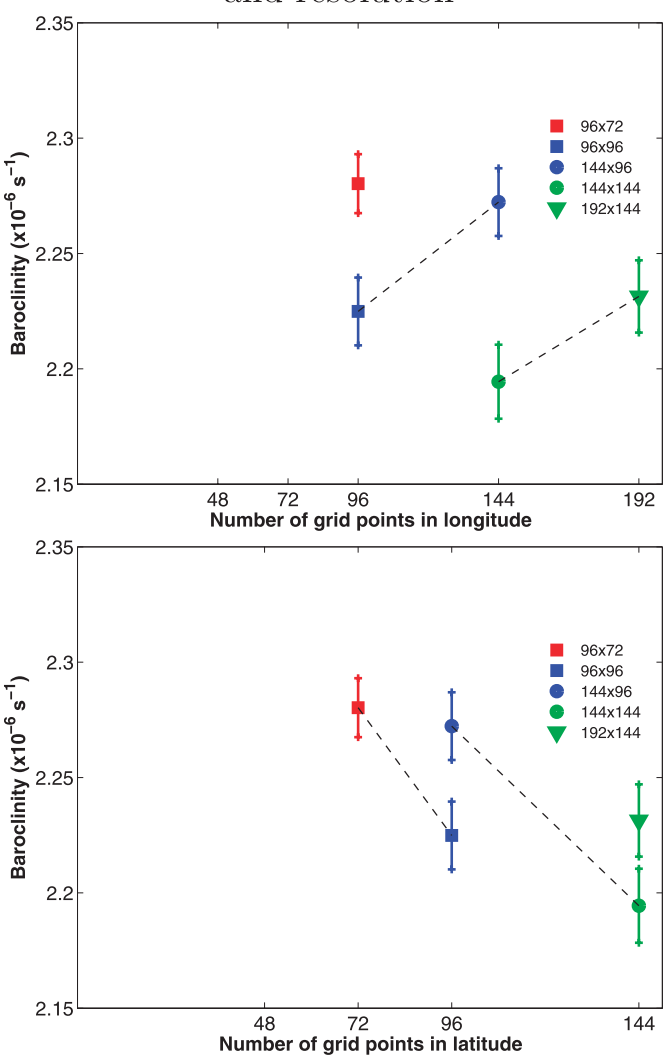

b) Relation between Ferrel cell strength and resolution
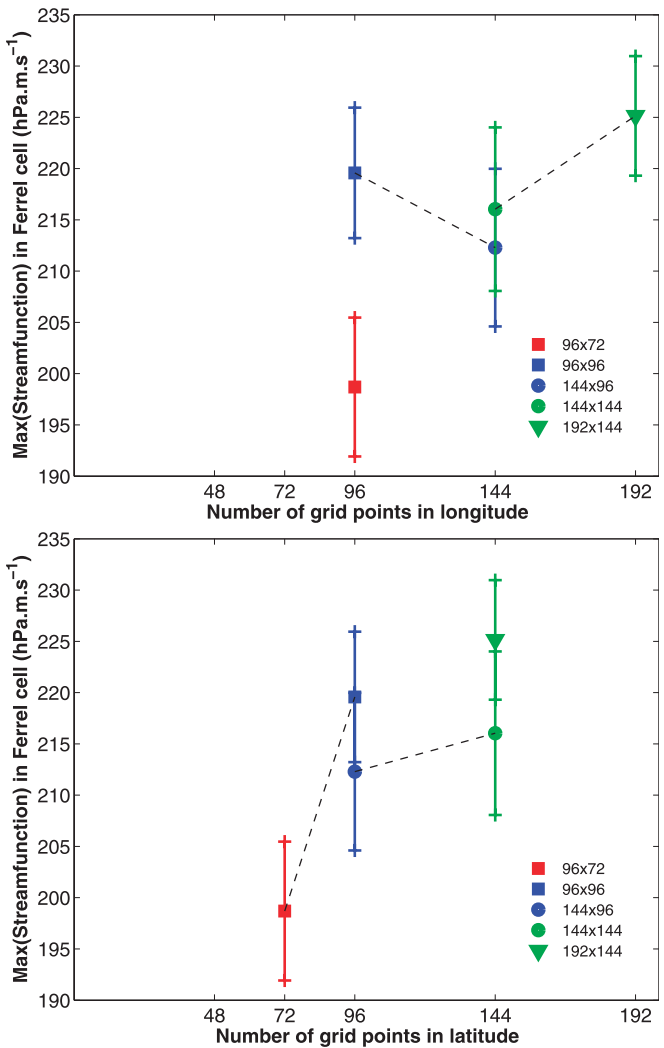

FIG. 9. Coupled simulations: (a) time-mean baroclinicity $\left(\mathrm{s}^{-1}\right)$ averaged between 200 and $1000 \mathrm{hPa}$ at the location of the jet; (b) strength of the Ferrel cell, defined as the maximum of the zonal-mean meridional streamfunction $\left(\mathrm{hPa} \mathrm{m} \mathrm{s}{ }^{-1}\right)$. Values are plotted as a function of the resolution in longitude (latitude), with dashed lines linking simulations with the same resolution in latitude (longitude) in top (bottom) panels. The $95 \%$ confidence intervals are given together with the mean value.

resolution increases, the Ferrel cell does not strengthen in the free idealized experiment (section 3b).

In the idealized experiments, the baroclinic wave sources increased with the resolution in longitude. The direct impacts on the zonal-mean state were exactly compensated in the free experiments by a strengthening of the Ferrel cell, resulting in no significant change in temperature gradient or vertical wind shear. Such compensation does not seem to occur in the full coupled model. Possible reasons could be the interaction with the ocean, which plays a strong role in setting the low-level temperature gradient, or the role or moist or other diabatic processes in the full atmospheric model. These processes could also explain the differences between the idealized and realistic experiments when increasing the resolution in latitude. However, a complete study is outside the scope of this paper.

\section{Conclusions}

In this study, we aimed to diagnose and understand the impact of the atmospheric horizontal resolution on the midlatitude atmospheric circulation in the LMDz (Hourdin et al. 2006) model, the atmospheric component of the IPSL coupled model. The most striking result is that although the midlatitude jet systematically shifts poleward when increasing the resolution in latitude, it does not move or even shift slightly equatorward when the increase is in longitude. This behavior is observed in a number of model configurations, from the coupled climate model to dynamical core simulations following the Held and Suarez (1994) idealized setup.

To explore further the different impacts of resolution changes and understand their origin, a series of additional experiments was conducted in the idealized setup 
in which the zonal-mean zonal wind was constrained to follow the one obtained in a reference simulation. Below are the results for an increase of the resolution in latitude and longitude:

1) Latitude-In the constrained experiments, the increase in low-level baroclinic wave source leads to enhanced poleward momentum transport across the jet when the waves propagate equatorward. In the unconstrained experiments, this leads to a poleward shift of the jet and of the associated regions of low-level baroclinicity and wave sources. However, the local baroclinicity becomes weaker, resulting in less energetic waves. The position of the zonalmean jet also becomes more variable in time.

2) Longitude-The increase in wave sources at the surface here is accompanied by a change of their behavior in the free troposphere, with a stronger tendency toward poleward propagation, and thus equatorward momentum transport, around the jet latitude. A poleward jet shift is thus inhibited, and the jet tends to remain in place. The larger lowlevel meridional heat flux by the waves is offset by a strengthening of the Ferrel cell, leading to no change in the baroclinicity.

A uniform increase in resolution leads to more energetic waves and a shift in latitude of the jet, consistent with previous studies. Our results suggest, however, that resolution changes in different directions are responsible for each effect.

An important conclusion of this study for modelers is the same one reached by Held and Phillipps (1993) in simulations at much lower resolutions: a grid with enhanced resolution in latitude might be better to simulate the large-scale midlatitude circulation, even at high resolution (we reached no convergence at resolutions typical of modern climate models). In the specific case of a latitude-longitude grid, an optimum could be to take the same number of points in both directions, giving an isotropic grid at $60^{\circ}$. The jet keeps moving poleward as the latitudinal resolution is increased even further, but the computational cost becomes higher because the time step then has to decrease to meet the CFL criterion.

Important questions remain, especially regarding the causes for the changes in the preferential direction of propagation of the waves: is it due to the particular numerical scheme used in the model or to a more general property? The answer will require simulations with different models and well-defined idealized test cases.

Acknowledgments. The authors especially wish to thank Marie-Alice Foujols, who ran the IPSL CM4 coupled experiments. Gwendal Riviere and Guillaume
Lapeyre are greatly acknowledged for the interesting discussions about our results. The authors are grateful to Gang Chen for his script to compute baroclinic wave spectra. This study was supported by the European Commission's Sixth Framework Programme (ENSEMBLES Contract GOCE-CT-2005-505539).

\section{REFERENCES}

Boer, G. J., and M. Lazare, 1988: Some results concerning the effect of horizontal resolution and gravity-wave drag on simulated climate. J. Climate, 1, 789-806.

__ and B. Denis, 1997: Numerical convergence of the dynamics of a GCM. Climate Dyn., 13, 359-374.

Boville, B. A., 1991: Sensitivity of simulated climate to model resolution. J. Climate, 4, 469-485.

Boyle, J. S., 1993: Sensitivity of dynamical quantities to horizontal resolution for a climate simulation using the ECMWF (cycle 33) model. J. Climate, 6, 796-815.

Branković, C., and D. Gregory, 2001: Impact of horizontal resolution on seasonal integrations. Climate Dyn., 18, 123-143.

Chen, G., I. M. Held, and W. A. Robinson, 2007: Sensitivity of the latitude of the surface westerlies to surface friction. J. Atmos. Sci., 64, 2899-2915.

Chen, M., R. B. Rood, and L. L. Takacs, 1997: Impact of a semiLagrangian and an Eulerian dynamical core on climate simulations. J. Climate, 10, 2374-2389.

Chen, T. C., and J. J. Tribbia, 1993: An effect of the model's horizontal resolution on stationary eddies simulated by the NCAR CCM1. J. Climate, 6, 1657-1664.

Déqué, M., C. Dreverton, A. Braun, and D. Cariolle, 1994: The ARPEGE/IFS atmosphere model: A contribution to the French community climate modelling. Climate Dyn., 10, 249-266.

Fichefet, T., and M. A. Morales Maqueda, 1997: Sensitivity of a global sea ice model to the treatment of ice thermodynamics and dynamics. J. Geophys. Res., 102, 12 609-12 646.

— , and - 1999: Modelling the influence of snow accumulation and snow-ice formation on the seasonal cycle of the Antarctic sea-ice cover. Climate Dyn., 15, 251-268.

Gerber, E. P., and G. K. Vallis, 2007: Eddy-zonal flow interactions and the persistence of the zonal index. J. Atmos. Sci., 64, 32963311.

_ - S. Voronin, and L. M. Polvani, 2008: Testing the annular mode autocorrelation time scale in simple atmospheric general circulation models. Mon. Wea. Rev., 136, 1523-1536.

Hack, J. J., J. M. Caron, G. Danabasoglu, K. W. Oleson, C. Bitz, and J. E. Truesdale, 2006: CCSM-CAM3 climate simulation sensitivity to changes in horizontal resolution. J. Climate, 19, 2267-2289.

Hamilton, K., R. J. Wilson, J. D. Mahlman, and L. J. Umschied, 1995: Climatology of the SKYHI troposphere-stratospheremesosphere general circulation model. J. Atmos. Sci., 52, 5-43.

Held, I. M., and P. J. Phillipps, 1993: Sensitivity of the eddy momentum flux to meridional resolution in atmospheric GCMs. J. Climate, 6, 499-506.

—_, and M. J. Suarez, 1994: A proposal of the dynamical cores of atmospheric general circulation models. Bull. Amer. Meteor. Soc., 75, 1825-1830.

Hourdin, F., and A. Armengaud, 1999: The use of finite-volume methods for atmospheric advection of trace species. Part I: Test of various formulations in a general circulation model. Mon. Wea. Rev., 127, 822-837. 
, and Coauthors, 2006: The LMDZ4 general circulation model: Climate performance and sensitivity to parametrized physics with emphasis on tropical convection. Climate Dyn., 27, 787-813.

Jablonowski, C., 1998: Test of the dynamics of two global weather prediction models of the German Weather Service: The HeldSuarez test (in German). M.S. thesis, Dept. of Meteorology, Meteorological Institute of the University of Bonn, $151 \mathrm{pp}$.

Kasahara, A., 1977: Computational aspects of numerical models for weather prediction and climate simulation. Methods in Computational Physics, J. Chang, Ed., Vol. 17, Academic Press, 1-66.

Kiehl, J. T., and D. L. Williamson, 1991: Dependence of cloud amount on horizontal resolution in the National Center for Atmospheric Research Community Climate Model. J. Geophys. Res., 96, 10 955-10 980.

Krinner, G., C. Genthon, Z. X. Li, and P. L. Van, 1997: Studies of the Antarctic climate with a stretched-grid general circulation model. J. Geophys. Res., 102, 13 731-13 745.

Lorenz, D. J., and D. L. Hartmann, 2001: Eddy-zonal flow feedback in the Southern Hemisphere. J. Atmos. Sci., 58, 33123327.

Madec, G., P. Delecluse, M. Imbard, and C. Lévy, 1997: OPA version 8.1 ocean general circulation model reference manual LODYC Tech. Rep. 3, 91 pp.

Marti, O., and Coauthors, 2005: The new IPSL climate system model: IPSL-CM4 model. L'Institut Pierre-Simon Laplace Note du Pôle de Modélisation 26, 84 pp.

- , and Coauthors, 2010: Key features of the IPSL ocean atmosphere model and its sensitivity to atmospheric resolution. Climate Dyn., 34, 1-26.

Orlanski, I., 2003: Bifurcation in eddy life cycles: Implications for storm track variability. J. Atmos. Sci., 60, 993-1023.

Phillips, T. J., L. C. Corsetti, and S. L. Grotch, 1995: The impact of horizontal resolution on moist processes in the ECMWF model. Climate Dyn., 11, 85-102.

Pope, V. D., and R. A. Stratton, 2002: The processes governing horizontal resolution sensitivity in a climate model. Climate Dyn., 19, 211-236.
Ringler, T., R. Heikes, and D. Randall, 2000: Modeling the atmospheric general circulation using a spherical geodesic grid: A new class of dynamical cores. Mon. Wea. Rev., 128, 2471-2490.

Robinson, W. A., 2006: On the self-maintenance of midlatitude jets. J. Atmos. Sci., 63, 2109-2122.

Roeckner, E., and Coauthors, 2006: Sensitivity of simulated climate to horizontal and vertical resolution in the ECHAM5 atmosphere model. J. Climate, 19, 3771-3791.

Russell, J. L., R. J. Stouffer, and K. W. Dixon, 2006: Intercomparison of the Southern Ocean circulations in IPCC coupled model control simulations. J. Climate, 19, 4560-4575.

Sadourny, R., 1975a: Compressible model flows on the sphere. J. Atmos. Sci., 32, 2103-2110.

, 1975b: The dynamics of finite-difference models of the shallow-water equations. J. Atmos. Sci., 32, 680-689.

Stendel, M. and E. Roeckner, 2006: Impacts of horizontal resolution on simulated climate statistics in ECHAM4. Max Planck Institute for Meteorology Rep. 253, 57 pp. [Available from MPI for Meteorology, Bundesstr. 53, D-20146 Hamburg, Germany.]

Stratton, R. A., 1999: A high resolution AMIP integration using the Hadley Centre model HadAM2b. Climate Dyn., 15, 9-28.

Van Leer, B., 1977: Towards the ultimate conservative difference scheme: IV. A new approach to numerical convection. J. Comput. Phys., 23, 276-299.

Wan, H., M. A. Giorgetta, and L. Bonaventura, 2008: Ensemble Held-Suarez test with a spectral transform model: Variability, sensitivity, and convergence. Mon. Wea. Rev., 136, 1075-1092.

Williamson, D. L., 1999: Convergence of atmospheric simulations with increasing horizontal resolution and fixed forced scales. Tellus, 51A, 663-673.

- 2008: Convergence of aqua-planet simulations with increasing resolution in the community atmospheric model, version 3. Tellus, $\mathbf{6 0 A}, 848-862$.

—, J. T. Kiehl, and J. J. Hack, 1995: Climate sensitivity of the NCAR Community Climate Model (CCM2) to horizontal resolution. Climate Dyn., 11, 377-397.

, J. G. Olson, and B. A. Boville, 1998: A comparison of semiLagrangian and Eulerian tropical climate simulations. Mon. Wea. Rev., 126, 1001-1012. 